\title{
A VIABILITY THEOREM FOR MORPHOLOGICAL INCLUSIONS*
}

\author{
THOMAS LORENZ ${ }^{\dagger}$
}

\begin{abstract}
The aim of this paper is to adapt the Viability Theorem from differential inclusions (governing the evolution of vectors in a finite dimensional space) to so-called morphological inclusions (governing the evolution of nonempty compact subsets of the Euclidean space).

In this morphological framework, the evolution of compact subsets of $\mathbb{R}^{N}$ is described by means of flows along bounded Lipschitz vector fields (similarly to the velocity method alias speed method in shape analysis). Now for each compact subset, more than just one vector field is admitted correspondingly to the set-valued map of a differential inclusion in finite dimensions.

We specify sufficient conditions on the given data such that for every initial compact set, at least one of these compact-valued evolutions satisfies fixed state constraints in addition. The proofs follow an approximative track similar to the standard approach for differential inclusions in $\mathbb{R}^{N}$, but they use tools about weak compactness and weak convergence of Banach-valued functions. Finally an application to shape optimization under state constraints is sketched.
\end{abstract}

Key words. Shape evolutions with state constraints, velocity method (speed method), morphological equations, Nagumo's theorem, viability condition, Clarke's generalized shape derivative.

AMS subject classifications. 49J53, 34A60, 47N10, 49J24, 49Q10, 93C15

1. Introduction. State constraints provide challenging questions in any form of dynamic system. Asking for sufficient and necessary conditions on the state constraints, the first complete answer for ordinary differential equations was given by Nagumo [26] in 1942 and, this characterization (using Bouligand tangent cones) has been rediscovered many times during the last decades.

If solutions of any given initial value problem are not unique, then two versions of this question are to be distinguished from each other: Either we demand all solutions to have their values in the fixed constrained set or (just) at least one solution with this property has to exist. In the first case, the corresponding constrained set is called invariant and, in the latter case, it is viable or weakly invariant. For autonomous differential inclusions in $\mathbb{R}^{N}$, the results are presented in Aubin's monography Viability theory [5], for example.

The main goal of this paper is a sufficient characterization of viability for shapes.

To be more precise, we leave the familiar Euclidean space $\mathbb{R}^{N}$ and consider evolutions of nonempty compact subsets of $\mathbb{R}^{N}$ instead. Correspondingly, the solution $x:[0, T] \longrightarrow \mathbb{R}^{N}$ (of a differential inclusion) is now replaced by a curve $K:[0, T] \longrightarrow \mathcal{K}\left(\mathbb{R}^{N}\right)$ with $\mathcal{K}\left(\mathbb{R}^{N}\right)$ denoting the set of nonempty compact subsets of $\mathbb{R}^{N}$ usually supplied with the Pompeiu-Hausdorff distance

$$
d\left(K_{1}, K_{2}\right):=\sup _{\substack{x \in K_{1} \\ y \in K_{2}}}\left\{\operatorname{dist}\left(x, K_{1}\right), \operatorname{dist}\left(y, K_{2}\right)\right\}=\sup _{z \in \mathbb{R}^{N}}\left|\operatorname{dist}\left(z, K_{1}\right)-\operatorname{dist}\left(z, K_{2}\right)\right| .
$$

The state constraints are again formulated as a subset, i.e. now $\mathcal{V} \subset \mathcal{K}\left(\mathbb{R}^{N}\right)$ (instead of $V \subset \mathbb{R}^{N}$ for differential inclusions).

*This work was supported by European Community's Human Potential Programme under contract HPRN-CT-2002-00281 [Evolution Equations].

$\dagger$ Interdisciplinary Center for Scientific Computing (IWR), Ruprecht-Karls-University of Heidelberg, Im Neuenheimer Feld 294, 69120 Heidelberg (Germany) thomas.lorenz@iwr.uni-heidelberg.de First preprint: August 11, 2006, this revised version: July 13, 2007

Submitted to SIAM Journal of Control and Optimization. 
Lipschitz vector fields for specifying time derivatives of curves in $\left(\mathcal{K}\left(\mathbb{R}^{N}\right), d\right)$

For formulating the viability problem in the metric space $\left(\mathcal{K}\left(\mathbb{R}^{N}\right), d\right)$, we have to specify how compact subsets of $\mathbb{R}^{N}$ are "deformed". The so-called velocity method or speed method has led Céa, Delfour, Zolésio and others to remarkable results about shape optimization (see e.g. $[9,11,12,33,38]$ and references there). It is based on prescribing a vector field $v: \mathbb{R}^{N} \times[0, T] \longrightarrow \mathbb{R}^{N}$ such that the corresponding ordinary differential equation $\frac{d}{d t} x(\cdot)=v(x(\cdot), \cdot)$ induces a unique flow on $\mathbb{R}^{N}$. Indeed, supposing $v$ to be sufficiently smooth, the Cauchy problem

$$
\frac{d}{d t} x(\cdot)=v(x(\cdot), \cdot) \text { in }[0, T], \quad x(0)=x_{0} \in \mathbb{R}^{N}
$$

is always well-posed and, any compact initial set $K \subset \mathbb{R}^{N}$ is deformed to

$$
\vartheta_{v}(t, K):=\left\{x(t) \mid \exists x(\cdot) \in C^{1}\left([0, t], \mathbb{R}^{N}\right): \frac{d}{d t} x(\cdot)=v(x(\cdot), \cdot) \text { in }[0, t], x(0) \in K\right\}
$$

after an arbitrary time $t \geq 0$. As a key advantage, this concept of set evolution does not require any regularity conditions on the compact set $K$ or its topological boundary (but only on the vector field $v$ ). In a word, $v$ can be interpreted as a "direction of deformation" for $\left(\mathcal{K}\left(\mathbb{R}^{N}\right), d l\right)$. So it is "possible to define directional derivatives and speak of shape gradient and shape Hessian with respect to the associated vector space of velocities. This [...] approach has been known in the literature as the velocity method' [11, Chapter $1, \S 6]$.

Aubin seized this notion for extending ODEs to this metric space of compact subsets. The so-called morphological equations are sketched in [4] and then presented in [2,3] in more detail. (They seem to be closer to ODEs in $\mathbb{R}^{N}$ than Panasyuk's concept of "quasidifferential equations" [27, 28, 29].)

For a given curve $K(\cdot):[0, T] \longrightarrow \mathcal{K}\left(\mathbb{R}^{N}\right)$, autonomous Lipschitz vector fields $\mathbb{R}^{N} \longrightarrow \mathbb{R}^{N}$ are used for specifying the counterparts of time derivatives. To be more precise, a Lipschitz continuous field $g: \mathbb{R}^{N} \longrightarrow \mathbb{R}^{N}$ represents a first-order approximation of $K(\cdot)$ at time $t \in[0, T[$ if

$$
\limsup _{h \downarrow 0} \frac{1}{h} \cdot d l\left(K(t+h), \quad \vartheta_{g}(h, K(t))\right)=0 .
$$

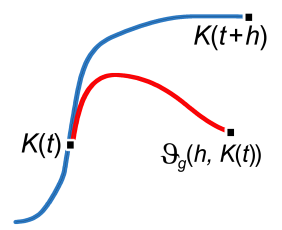

Obviously, this limit superior being equal to 0 is even a limit because distances are always nonnegative by definition. Of course, such a field $g(\cdot)$ need not be unique and thus, all bounded Lipschitz vector fields with this property $(*)$ form the so-called mutation $\stackrel{\circ}{K}(t)$ of $K(\cdot)$ at time $t \in[0, T[$. In particular, the mutation is a subset of all bounded Lipschitz functions $\mathbb{R}^{N} \longrightarrow \mathbb{R}^{N}$ and extends the time derivative to curves in the metric space $\left(\mathcal{K}\left(\mathbb{R}^{N}\right), d l\right)$.

Solving a morphological equation with state constraints:

Aubin's adaptation of Nagumo's theorem

The step from specifying a time derivative (of a curve) to formulating a (generalized) differential equation is rather small. It is based just on prescribing the time derivative as a function of the current state. In connection with nonempty compact subsets of $\mathbb{R}^{N}$, a function $f: \mathcal{K}\left(\mathbb{R}^{N}\right) \longrightarrow \operatorname{Lip}\left(\mathbb{R}^{N}, \mathbb{R}^{N}\right)$ is given with $\operatorname{Lip}\left(\mathbb{R}^{N}, \mathbb{R}^{N}\right)$ denoting the set of all bounded and Lipschitz continuous functions $\mathbb{R}^{N} \longrightarrow \mathbb{R}^{N}$. 
For any initial set $K_{0} \in \mathcal{K}\left(\mathbb{R}^{N}\right)$, we are looking for $K(\cdot):[0, T] \longrightarrow \mathcal{K}\left(\mathbb{R}^{N}\right)$ satisfying 1. $K(\cdot)$ is Lipschitz continuous with respect to the Pompeiu-Hausdorff distance $d$,

2. $f(K(t)) \in \stackrel{\circ}{K}(t)$ for a.e. $t \in\left[0, T\right.$, i.e. $\lim _{h \downarrow 0} \frac{1}{h} \cdot d l\left(K(t+h), \vartheta_{f(K(t))}(h, K(t))\right)=0$,

3. $K(0)=K_{0}$.

Then, $K(\cdot)$ is called solution of the (autonomous) morphological equation $\stackrel{\circ}{K}(\cdot) \ni$ $f(K(\cdot))$ in $[0, T]$ with initial value $K_{0}$.

At first glance, the symbol $\ni$ here seems to be contradictory to the term "equation". The mutation $\stackrel{\circ}{K}(t)$, however, is defined as subset of all transitions providing a firstorder approximation of $K(t+\cdot)$ and so, the "right-hand side" $f(K(t)) \in \operatorname{Lip}\left(\mathbb{R}^{N}, \mathbb{R}^{N}\right)$ should be one of its elements. (In the classical framework of differentiable functions and vector spaces, the mutation consists of just one vector.)

Considering now additional state constraints, the question about existence of a solution has been answered completely by Aubin in [3, Theorem 0.1]. In particular, the assumptions about state constraints and $f(\cdot)$ justify its interpretation as a counterpart of Nagumo's theorem. Some applications and further studies are presented in $[16,18,22]$.

Proposition 1.1 (Nagumo's theorem for morphological equations $[2,3]$ ). Suppose $\mathcal{V} \subset \mathcal{K}\left(\mathbb{R}^{N}\right)$ to be nonempty and closed with respect to $\mathrm{d}$. Let $f:\left(\mathcal{K}\left(\mathbb{R}^{N}\right), d l\right) \longrightarrow\left(\operatorname{Lip}\left(\mathbb{R}^{N}, \mathbb{R}^{N}\right),\|\cdot\|_{\infty}\right)$ be a continuous function satisfying

1. uniform bound of Lipschitz constants: $\sup _{M \in \mathcal{K}\left(\mathbb{R}^{N}\right)} \operatorname{Lip} f(M)<\infty$, 2. uniform bound of supremum norms: $\sup _{M \in \mathcal{K}\left(\mathbb{R}^{N}\right)}\|f(M)\|_{\infty}<\infty$.

For any $M \in \mathcal{V}$, let $f(M) \in \operatorname{Lip}\left(\mathbb{R}^{N}, \mathbb{R}^{N}\right)$ be contingent to $\mathcal{V}$ at $M$ in the sense that

$$
\begin{aligned}
0= & \liminf _{h \downarrow 0} \frac{1}{h} \cdot \operatorname{dist}\left(\vartheta_{f(M)}(h, M), \mathcal{V}\right) \\
& \stackrel{\text { Def. }}{=} \liminf _{h \downarrow 0} \frac{1}{h} \cdot \inf _{C \in \mathcal{V}} d\left(\vartheta_{f(M)}(h, M), C\right) .
\end{aligned}
$$

Then, from any $K_{0} \in \mathcal{V}$ starts a solution $K(\cdot):\left[0, \infty\left[\longrightarrow \mathcal{K}\left(\mathbb{R}^{N}\right)\right.\right.$ of the morphological equation $\stackrel{\circ}{K}(\cdot) \ni f(K(\cdot))$ which is viable in $\mathcal{V}$, i.e. $K(t) \in \mathcal{V}$ for all $t$.

The new step to morphological inclusions

This paper focuses on the corresponding conditions (of viability) if more than one Lipschitz field is admitted for each compact set, i.e. the single-valued function $f$ : $\mathcal{K}\left(\mathbb{R}^{N}\right) \longrightarrow \operatorname{Lip}\left(\mathbb{R}^{N}, \mathbb{R}^{N}\right)$ is replaced by a set-valued map $\mathcal{F}: \mathcal{K}\left(\mathbb{R}^{N}\right) \rightsquigarrow \operatorname{Lip}\left(\mathbb{R}^{N}, \mathbb{R}^{N}\right)$. This modification of given data leads directly to the following definition: A Lipschitz continuous curve $K(\cdot):[0, T] \longrightarrow\left(\mathcal{K}\left(\mathbb{R}^{N}\right), d l\right)$ is called solution of the morphological inclusion

$$
\stackrel{\circ}{K}(\cdot) \cap \mathcal{F}(K(\cdot)) \neq \emptyset \quad \text { in }[0, T[
$$

if $\mathcal{F}(K(t)) \cap \stackrel{\circ}{K}(t) \neq \emptyset$ for almost every $t \in[0, T[$, i.e. there exists $w \in \mathcal{F}(K(t)) \subset$ $\operatorname{Lip}\left(\mathbb{R}^{N}, \mathbb{R}^{N}\right)$ with $\lim _{h \downarrow 0} \frac{1}{h} \cdot d\left(K(t+h), \vartheta_{w}(h, K(t))\right)=0$.

Obviously, every morphological equation can be regarded as a morphological inclusion (just with single-valued $\mathcal{F}$ ). So this step provides a real extension. 
Considering now additional state constraints on $K(\cdot)$, Doyen [19] has given sufficient and some necessary conditions on $\mathcal{F}(\cdot)$ and $\mathcal{V} \subset \mathcal{K}\left(\mathbb{R}^{N}\right)$ for the invariance of $\mathcal{V}$ (i.e. all continuous solutions starting in $\mathcal{V}$ stay in $\mathcal{V}$ ). His key notion is first to extend Filippov's existence theorem from differential inclusions (in $\mathbb{R}^{N}$ ) to morphological inclusions in $\mathcal{K}\left(\mathbb{R}^{N}\right)[19$, Theorem 7.1] and then to verify $\operatorname{dist}(K(\cdot), \mathcal{V}) \leq 0$ (under the assumption that the values of $\mathcal{F}(\cdot)$ are always contained in the corresponding contingent cone to $\mathcal{V})[19$, Theorem 8.2].

The main result here concerns sufficient conditions on $\mathcal{F}(\cdot)$ and $\mathcal{V} \subset \mathcal{K}\left(\mathbb{R}^{N}\right)$ for the viability of $\mathcal{V}$, i.e. at least one Lipschitz continuous solution has to stay in $\mathcal{V}$. This question (in a more general environment) was pointed out as open in [2, § 2.3.3] and, to the best of my knowledge, it has not been answered even for the special case of morphological inclusions so far.

In fact, the following statement is very similar to the viability theorem for differential inclusions in $\mathbb{R}^{N}$ (as it is discussed in [5, Theorems 3.3.2, 3.3.4] and quoted here in Theorem 3.3). Roughly speaking, $\mathcal{F}$ is supposed to be upper semicontinuous with closed convex values - after specifying a suitable topology on $\operatorname{Lip}\left(\mathbb{R}^{N}, \mathbb{R}^{N}\right)$ in a moment - and, we require (at least) one "contingent direction" in the value $\mathcal{F}(K) \subset \operatorname{Lip}\left(\mathbb{R}^{N}, \mathbb{R}^{N}\right)$ for each $K \in \mathcal{V}$.

THEOREM 1.2 (Viability theorem for morphological inclusions).

Let $\mathcal{F}: \mathcal{K}\left(\mathbb{R}^{N}\right) \rightsquigarrow \operatorname{Lip}\left(\mathbb{R}^{N}, \mathbb{R}^{N}\right)$ be a set-valued map and $\mathcal{V} \subset \mathcal{K}\left(\mathbb{R}^{N}\right)$ a nonempty closed subset satisfying:

1.) all values of $\mathcal{F}$ are nonempty and convex (i.e. for any $\lambda \in[0,1]$ and

$g_{1}, g_{2} \in \mathcal{F}(K) \subset \operatorname{Lip}\left(\mathbb{R}^{N}, \mathbb{R}^{N}\right)$, the function $\lambda \cdot g_{1}+(1-\lambda) \cdot g_{2} \in \operatorname{Lip}\left(\mathbb{R}^{N}, \mathbb{R}^{N}\right)$

also belongs to $\mathcal{F}(K)$ ),

2.) $\sup _{M \in \mathcal{K}\left(\mathbb{R}^{N}\right)} \sup _{f \in \mathcal{F}(M)} \operatorname{Lip} f<\infty \quad$ (uniformly bounded Lipschitz constants),

$M \in \mathcal{K}\left(\mathbb{R}^{N}\right) \quad f \in \mathcal{F}(M)$

$\sup _{\mathcal{L}\left(\mathbb{R}^{N}\right)} \sup _{f \in \mathcal{F}(M)}\|f\|_{\infty}<\infty \quad$ (uniformly bounded sup norms),

3.) the graph of $\mathcal{F}$ is closed (w.r.t. locally uniform convergence in $\operatorname{Lip}\left(\mathbb{R}^{N}, \mathbb{R}^{N}\right)$ ),

4.) for each $K \in \mathcal{V}$, some $w \in \mathcal{F}(K) \subset \operatorname{Lip}\left(\mathbb{R}^{N}, \mathbb{R}^{N}\right)$ is contingent to $\mathcal{V}$ at $K$

in the sense that $\quad 0=\liminf _{h \downarrow 0} \frac{1}{h} \cdot \operatorname{dist}\left(\vartheta_{w}(h, K), \mathcal{V}\right)$.

Then for every initial compact set $K_{0} \in \mathcal{V}$, there exists at least one solution $K(\cdot):[0,1] \longrightarrow \mathcal{K}\left(\mathbb{R}^{N}\right)$ of the morphological inclusion $\stackrel{\circ}{K}(\cdot) \cap \mathcal{F}(K(\cdot)) \neq \emptyset$ with $K(0)=K_{0}$ and $K(t) \in \mathcal{V}$ for all $t \in[0,1]$.

The new analytical aspects are closely related to the proof of this theorem. Indeed, Haddad and others realized the theorem of Alaoglu as a powerful tool for constructing solutions of differential inclusions in $\mathbb{R}^{N}$ under state constraints. The counterparts of time derivatives here, however, form a bounded sequence in $L^{\infty}\left([0,1], \operatorname{Lip}\left(\mathbb{R}^{N}, \mathbb{R}^{N}\right)\right)$ which cannot be identified with a dual space in an obvious way. So results of Ülger and Kisielewicz come now into play for characterizing weakly compact subsets of the Bochner integrable functions $[0,1] \longrightarrow X$ (denoted by $L^{1}([0,1], X)$ ) and the set $C^{0}(K, X)$ of continuous functions $K \longrightarrow X$ with a real Banach space $X$ and a nonempty compact set $K \subset \mathbb{R}^{N}[23,34]$. 
Sketching an application to shape optimization under state constraints

In shape optimization, the aim is to detect a minimizer of a given shape functional $J: \mathcal{K}\left(\mathbb{R}^{N}\right) \longrightarrow \mathbb{R}$. An additional constrained set $\left.\mathcal{V} \subset \mathcal{K}\left(\mathbb{R}^{N}\right)\right)$ makes the problem rather complicated in general.

As an application of our Viability Theorem 1.2, we suggest a set-valued map $\mathcal{F}$ : $\mathcal{K}\left(\mathbb{R}^{N}\right) \rightsquigarrow \operatorname{Lip}\left(\mathbb{R}^{N}, \mathbb{R}^{N}\right.$ ) (by means of generalized shape derivatives of $J$ ) with the objective that every solution $K(\cdot):\left[0, \infty\left[\longrightarrow \mathcal{K}\left(\mathbb{R}^{N}\right)\right.\right.$ of the morphological inclusion $\stackrel{\circ}{K}(\cdot) \cap \mathcal{F}(K(\cdot)) \neq \emptyset$ satisfies the following two conditions and thus provides candidates (for the wanted minimizer):

(i) $\quad t \mapsto J(K(t))$ is nonincreasing and

(ii) every compact set $C=\operatorname{Lim}_{n \rightarrow \infty} K\left(t_{n}\right) \in \mathcal{V}$ (for some sequence $t_{n} \nearrow \infty$ )

satisfies a necessary condition on minimizers (in the form of Fermat's rule).

Then Viability Theorem 1.2 provides sufficient conditions on $\mathcal{F}$ and $\mathcal{V}$ for the existence of at least one solution $K(\cdot)$ with all its values in $\mathcal{V}$ (see Proposition 4.6).

This introduction $(\S 1)$ is reflecting the structure of the paper: Aubin's theory of morphological equations is summarized in $\S 2$. In particular, we mention the counterparts of Filippov's and Nagumo's theorems for evolutions in the metric space $\left(\mathcal{K}\left(\mathbb{R}^{N}\right), d l\right)$. Then, $\S 3$ provides the step to morphological inclusions. It starts with the viability theorem about differential inclusions (in $\S 3.1$ ), collects the tools for Banach-valued functions (in $\S 3.2$ ) and verifies the viability theorem for morphological inclusions (in $\S 3.3$ ). Finally, in $\S 4$, we present the analytical details of the application to shape optimization.

2. A brief introduction to morphological equations. Morphological equations provide typical geometric examples of so-called mutational equations. First presented in [4] and elaborated in [3, 2], mutational equations are to extend ordinary differential equations to a metric space $(E, d)$. In a word, the key idea is to describe derivatives by means of continuous maps (called transitions) $\vartheta:[0,1] \times E \longrightarrow E$, $(h, x) \longmapsto \vartheta(h, x)$ instead of affine-linear maps $(h, x) \longmapsto x+h v$ (that are usually used in vector spaces). Strictly speaking, such a transition specifies the point $\vartheta(t, x) \in E$ to which any initial point $x \in E$ has been moved after time $t \in[0,1]$. It can be interpreted as a first-order approximation of a curve $\xi:[0, T[\longrightarrow$ at time $t \in[0, T[$ if

$$
\lim _{h \downarrow 0} \frac{1}{h} \cdot d(\xi(t+h), \vartheta(h, \xi(t)))=0 .
$$

The so-called morphological equations apply this concept to the set $\mathcal{K}\left(\mathbb{R}^{N}\right)$ of nonempty compact subsets of $\mathbb{R}^{N}$ supplied with the Pompeiu-Hausdorff distance $d$,

$$
\begin{aligned}
d l\left(K_{1}, K_{2}\right) & :=\sup _{\substack{x \in K_{1} \\
y \in K_{2}}}\left\{\operatorname{dist}\left(x, K_{1}\right), \operatorname{dist}\left(y, K_{2}\right)\right\} \\
& =\inf \left\{\rho>0 \mid K_{1} \subset K_{2}+\rho \mathbb{B}_{1}, K_{2} \subset K_{1}+\rho \mathbb{B}_{1}\right\} .
\end{aligned}
$$

Here $\mathbb{B}_{1}$ always denotes the closed unit ball in $\mathbb{R}^{N}$, i.e. $\mathbb{B}_{1}:=\left\{x \in \mathbb{R}^{N}|| x \mid \leq 1\right\}$. This is a very general starting point for geometric evolution problems as there are no a priori restriction in regard to the regularity of sets and their boundaries. Motivated by the velocity method (often used in shape optimization, e.g. [9, 11, 12, 33, 38]), ordinary differential equations are here to lay the basis for transitions. 
Definition 2.1. $\quad \operatorname{Lip}\left(\mathbb{R}^{N}, \mathbb{R}^{N}\right)$ consists of all bounded and Lipschitz continuous functions $\mathbb{R}^{N} \longrightarrow \mathbb{R}^{N}$.

Definition 2.2. Choosing any function $f: \mathbb{R}^{N} \times[0, T] \longrightarrow \mathbb{R}^{N}$, the so-called reachable set $\vartheta_{f}(t, K)$ of the initial set $K \in \mathcal{K}\left(\mathbb{R}^{N}\right)$ at time $t \in[0, T]$ is defined as

$$
\begin{aligned}
\vartheta_{f}(t, K):=\left\{x(t) \in \mathbb{R}^{N} \mid \begin{array}{l}
\exists x(\cdot) \in W^{1,1}\left([0, t], \mathbb{R}^{N}\right): x(0) \in K, \\
\left.\frac{d}{d \tau} x(\tau)=f(x(\tau), \tau) \text { for almost every } \tau \in[0, t]\right\}
\end{array}\right.
\end{aligned}
$$

(and correspondingly for an autonomous function $f: \mathbb{R}^{N} \longrightarrow \mathbb{R}^{N}$ ).

The special case of constant functions $f(\cdot) \equiv v$ (with an arbitrary vector $v \in \mathbb{R}^{N}$ ) leads to the Minkowski sum $\vartheta_{f}(t, K)=K+h \cdot v \subset \mathbb{R}^{N}$ and, for an initial set $K=\{x\}$ with just one element, in particular, we return to the familiar affine-linear map $(h, x) \longmapsto x+h \cdot v$ that has already been mentioned as motivation.

An essential contribution of Aubin was to specify appropriate continuity conditions on the maps $\vartheta:[0,1] \times E \longrightarrow E,(h, x) \longmapsto \vartheta(h, x)$ so that the familiar track of ordinary differential equations can be followed in a metric space $(E, d)$. Here we quote his definition introduced in the monograph [2] (emphasizing the local features slightly more than his original version in [3]). Reachable sets of every function $f \in \operatorname{Lip}\left(\mathbb{R}^{N}, \mathbb{R}^{N}\right)$ satisfy these conditions in the metric space $\left(\mathcal{K}\left(\mathbb{R}^{N}\right), d\right)$ :

Definition 2.3 ([2, Definition 1.1.2]). Let $(E, d)$ be a metric space. A map $\vartheta:[0,1] \times E \longrightarrow E$ is called transition on $(E, d)$ if it satisfies the following conditions:

1. $\vartheta(0, x)=x \quad$ for all $x \in E$,

2. $\lim _{h \downarrow 0} \frac{1}{h} \cdot d(\vartheta(t+h, x), \vartheta(h, \vartheta(t, x)))=0 \quad$ for all $x \in E, t \in[0,1[$,

3. $\alpha(\vartheta):=\max \left(0, \sup _{x \neq y} \limsup _{h \downarrow 0} \frac{d(\vartheta(h, x), \vartheta(h, y))-d(x, y)}{h \cdot d(x, y)}\right)<\infty$

4. $\beta(\vartheta):=\sup _{x \in E} \limsup _{h \downarrow 0} \frac{1}{h} \cdot d(x, \vartheta(h, x))<\infty$.

For any two transitions $\vartheta_{1}, \vartheta_{2}:[0,1] \times E \longrightarrow E$ on the same metric space $(E, d)$, the transitional distance between $\vartheta_{1}$ and $\vartheta_{2}$ is defined by

$$
d_{\Lambda}\left(\vartheta_{1}, \vartheta_{2}\right):=\sup _{x \in E} \limsup _{h \downarrow 0} \frac{1}{h} \cdot d\left(\vartheta_{1}(h, x), \vartheta_{2}(h, x)\right)
$$

Lemma 2.4. For every $f \in \operatorname{Lip}\left(\mathbb{R}^{N}, \mathbb{R}^{N}\right)$, the map $\vartheta_{f}:[0,1] \times \mathcal{K}\left(\mathbb{R}^{N}\right) \longrightarrow \mathcal{K}\left(\mathbb{R}^{N}\right)$, $(h, K) \longmapsto \vartheta_{f}(h, K)$ of reachable sets (as introduced in Definition 2.2) is a well-defined transition on the metric space $\left(\mathcal{K}\left(\mathbb{R}^{N}\right), d\right)$ according to Definition 2.3.

To be more precise, the reachable sets satisfy for all initial sets $K, K_{1}, K_{2} \in \mathcal{K}\left(\mathbb{R}^{N}\right)$, vector fields $f, g \in \operatorname{Lip}\left(\mathbb{R}^{N}, \mathbb{R}^{N}\right)$ and times $t, h \geq 0$

$$
\begin{aligned}
\vartheta_{f}(0, K) & =K, \\
\vartheta_{f}(t+h, K) & =\vartheta_{f}\left(h, \vartheta_{f}(t, K)\right), \\
d\left(\vartheta_{f}\left(h, K_{1}\right), \quad \vartheta_{f}\left(h, K_{2}\right)\right) & \leq d\left(K_{1}, K_{2}\right) \cdot e^{\operatorname{Lip} f \cdot h} \\
d l\left(\vartheta_{f}(h, K), \quad \vartheta_{g}(h, K)\right) & \leq\|f-g\|_{\infty} \cdot h e^{\operatorname{Lip} f \cdot h} \\
d\left(\vartheta_{f}(t, K), \vartheta_{f}(t+h, K)\right) & \leq\|f\|_{\infty} h
\end{aligned}
$$

and thus, $\alpha\left(\vartheta_{f}\right) \leq \operatorname{Lip} f, \beta\left(\vartheta_{f}\right) \leq\|f\|_{\infty}, d_{\Lambda}\left(\vartheta_{f}, \vartheta_{g}\right) \leq\|f-g\|_{\infty} \stackrel{\text { Def. }}{=} \sup _{x \in \mathbb{R}^{N}}|f(x)-g(x)|$. In particular, $\quad d l\left(\vartheta_{f}\left(h, K_{1}\right), \vartheta_{g}\left(h, K_{2}\right)\right) \leq e^{\operatorname{Lip} f \cdot h}\left(d l\left(K_{1}, K_{2}\right)+h \cdot\|f-g\|_{\infty}\right)$. 
The proof is presented in [2, Proposition 3.5.3] - as a consequence of Cauchy-Lipschitz Theorem (about solutions of ordinary differential equations). In particular, this lemma justifies calling $\vartheta_{f}$ a shape transition on $\left(\mathcal{K}\left(\mathbb{R}^{N}\right), d\right.$ ) (or morphological transition - in accordance with [2, Definition 3.7.2]). For the sake of simplicity, $f \in$ $\operatorname{Lip}\left(\mathbb{R}^{N}, \mathbb{R}^{N}\right)$ is sometimes identified with its shape transition $\vartheta_{f}$.

These reachable sets provide the tools for specifying (generalized) shape derivatives of a compact-valued tube $K(\cdot):\left[0, T\left[\rightsquigarrow \mathbb{R}^{N}\right.\right.$, i.e. a curve $K(\cdot):\left[0, T\left[\longrightarrow \mathcal{K}\left(\mathbb{R}^{N}\right)\right.\right.$. So the next step will be to solve equations prescribing an element of the shape mutation.

Definition $2.5([2, \S 3.5 .4])$. For any compact-valued tube $K(\cdot):\left[0, T\left[\rightsquigarrow \mathbb{R}^{N}\right.\right.$, the shape mutation $\stackrel{\circ}{K}(t)$ at time $t \in\left[0, T\right.$ [ consists of all $f \in \operatorname{Lip}\left(\mathbb{R}^{N}, \mathbb{R}^{N}\right)$ satisfying

$$
\lim _{h \downarrow 0} \frac{1}{h} \cdot d l\left(\vartheta_{f}(h, K(t)), K(t+h)\right)=0 .
$$

Definition 2.6. For any given function $f: \mathcal{K}\left(\mathbb{R}^{N}\right) \times\left[0, T\left[\longrightarrow \operatorname{Lip}\left(\mathbb{R}^{N}, \mathbb{R}^{N}\right)\right.\right.$, a compact-valued $K(\cdot):\left[0, T\left[\rightsquigarrow \mathbb{R}^{N}\right.\right.$ is called solution of the morphological equation

$$
\stackrel{\circ}{K}(\cdot) \ni f(K(\cdot), \cdot)
$$

if 1. $K(\cdot):\left[0, T\left[\rightsquigarrow \mathbb{R}^{N}\right.\right.$ is Lipschitz continuous with respect to $d l$ and

2. for almost every $t \in\left[0, T\left[, f(K(t), t) \in \operatorname{Lip}\left(\mathbb{R}^{N}, \mathbb{R}^{N}\right)\right.\right.$ belongs to $\stackrel{\circ}{K}(t)$ or, equivalently, $\quad \lim _{h \downarrow 0} \frac{1}{h} \cdot d l\left(\vartheta_{f(K(t), t)}(h, K(t)), K(t+h)\right)=0$.

These conditions on a solution are in accordance with [2, Definition 1.3.1] being formulated for the autonomous case (i.e. $f$ not depending on time explicitly).

As an essential result of $[2,3]$, the Euler algorithm can be applied in the framework of morphological equations and so, the Cauchy-Lipschitz Theorem (about ordinary differential equations) has the following counterpart that is proved in [2, Theorem 4.1.2] for the more general case that the values of $f$ are bounded Lipschitz continuous set-valued maps:

Theorem 2.7. $\quad$ Suppose $f:\left(\mathcal{K}\left(\mathbb{R}^{N}\right), d l\right) \longrightarrow\left(\operatorname{Lip}\left(\mathbb{R}^{N}, \mathbb{R}^{N}\right),\|\cdot\|_{\infty}\right)$ to be Lipschitz continuous with Lipschitz constant $\lambda$ and $M:=\sup _{K \in \mathcal{K}\left(\mathbb{R}^{N}\right)} \operatorname{Lip} f(K)<\infty$.

For every initial set $K_{0} \in \mathcal{K}\left(\mathbb{R}^{N}\right)$ and time $T>0$, there exists a unique compactvalued solution $K(\cdot):\left[0, T\left[\rightsquigarrow \mathbb{R}^{N}\right.\right.$ of the morphological equation $\stackrel{\circ}{K}(\cdot) \ni f(K(\cdot))$ with $K(0)=K_{0}$.

Furthermore every Lipschitz compact-valued tube $Q:\left[0, \infty\left[\rightsquigarrow \mathbb{R}^{N}\right.\right.$ with $\stackrel{\circ}{Q}(t) \neq \emptyset$ for every $t \geq 0$ satisfies the following estimate at each time $t \geq 0$

$d(K(t), Q(t)) \leq d l\left(K_{0}, Q(0)\right) \cdot e^{(M+\lambda) t}+\int_{0}^{t} e^{(M+\lambda)(t-s)} \cdot \inf _{g \in \grave{Q}(s)}\|f(Q(s))-g\|_{\infty} d s$. In particular, the solution $K(\cdot)$ depends on the initial set $K_{0}$ and the right-hand side $f$ in a Lipschitz continuous way.

Existence under (additional) state constraints proves to be a very interesting question for many applications. In the particular case of ordinary differential equations, Nagumo's Theorem gives a necessary and sufficient condition on the constrained set $\mathcal{V}$ for existence of local solutions. It uses the contingent cone (in the sense of Bouligand) and has served as a key motivation for viability theory (see e.g. [5]). 
Definition 2.8 ([5, Definition 1.1.3]). Let $X$ be a normed vector space, $V \subset X$ nonempty and $x \in V$. The contingent cone to $V$ at $x$ (in the sense of Bouligand) is

$$
T_{V}(x):=\left\{u \in X \mid \liminf _{h \downarrow 0} \frac{1}{h} \cdot \operatorname{dist}(x+h u, V)=0\right\} .
$$

This classical definition of contingent cone in a vector space is now extended to the metric space $\left(\mathcal{K}\left(\mathbb{R}^{N}\right), d\right)$ by using the shape transitions of $\operatorname{Lip}\left(\mathbb{R}^{N}, \mathbb{R}^{N}\right)$ :

$$
\begin{aligned}
& \text { Definition } 2.9\left(\left[2, \text { Definition 1.5.2]). For nonempty } \mathcal{V} \subset \mathcal{K}\left(\mathbb{R}^{N}\right) \text { and } K \in \mathcal{V},\right.\right. \\
& \begin{aligned}
T_{\mathcal{V}}(K):=\left\{f \in \operatorname{Lip}\left(\mathbb{R}^{N}, \mathbb{R}^{N}\right) \mid 0\right. & =\liminf _{h \downarrow 0} \frac{1}{h} \cdot \operatorname{dist}\left(\vartheta_{f}(h, K), \mathcal{V}\right) \\
& \left.\stackrel{\text { Def. }}{=} \liminf _{h \downarrow 0} \frac{1}{h} \cdot \inf _{C \in \mathcal{V}} d\left(\vartheta_{f}(h, K), C\right)\right\}
\end{aligned}
\end{aligned}
$$

is called contingent transition set of $\mathcal{V}$ at $K$.

The "geometric" background of reachable sets implies an additional property of shape transitions in $T_{\mathcal{V}}(K) \subset \operatorname{Lip}\left(\mathbb{R}^{N}, \mathbb{R}^{N}\right)$. Indeed, for any $f \in T_{\mathcal{V}}(K)$, every function $g \in \operatorname{Lip}\left(\mathbb{R}^{N}, \mathbb{R}^{N}\right)$ with $f(\cdot)=g(\cdot)$ in a neighborhood of $\partial K$ is also contained in $T_{\mathcal{V}}(K)$ because the Cauchy-Lipschitz Theorem about ODEs ensures $\partial \vartheta_{f}(t, K)=$ $\vartheta_{f}(t, \partial K)=\vartheta_{g}(t, \partial K)=\partial \vartheta_{g}(t, K)$ for small $t \geq 0$. So in other words, the criterion of $T_{\mathcal{V}}(K)$ depends only on an arbitrarily small neighborhood of the boundary $\partial K$.

In fact, Nagumo's Theorem also holds for morphological equations as shown in [2, Theorem 4.1.7] (again for the more general case that $f$ is a single-valued function whose values are uniformly bounded Lipschitz continuous set-valued maps $\mathbb{R}^{N} \rightsquigarrow \mathbb{R}^{N}$ ):

THEOREM 2.10 (Nagumo's theorem for morphological equations [2]). Suppose $\mathcal{V} \subset \mathcal{K}\left(\mathbb{R}^{N}\right)$ to be nonempty and closed with respect to dl. Let $f:\left(\mathcal{K}\left(\mathbb{R}^{N}\right), d l\right) \longrightarrow\left(\operatorname{Lip}\left(\mathbb{R}^{N}, \mathbb{R}^{N}\right),\|\cdot\|_{\infty}\right)$ be a continuous function satisfying

1. $\sup _{M \in \mathcal{K}\left(\mathbb{R}^{N}\right)} \operatorname{Lip} f(M)<\infty$,

2. $\sup _{M \in \mathcal{K}\left(\mathbb{R}^{N}\right)}\|f(M)\|_{\infty}<\infty$.

Then from any initial state $K_{0} \in \mathcal{V}$ starts at least one Lipschitz solution $K(\cdot)$ : $\left[0, T\left[\longrightarrow \mathcal{K}\left(\mathbb{R}^{N}\right)\right.\right.$ of $\stackrel{\circ}{K}(\cdot) \ni f(K(\cdot))$ viable in $\mathcal{V}($ i.e. $K(t) \in \mathcal{V}$ for all $t)$ if and only if $\mathcal{V}$ is a viability domain of $f$ in the sense of $f(M) \in T_{\mathcal{V}}(M)$ for each $M \in \mathcal{V}$.

3. The step to morphological inclusions. The main aim now is to prove a similar viability theorem for morphological inclusions, i.e. the single-valued function $f: \mathcal{K}\left(\mathbb{R}^{N}\right) \longrightarrow \operatorname{Lip}\left(\mathbb{R}^{N}, \mathbb{R}^{N}\right)$ of the right-hand side is to be replaced by a set-valued map $\mathcal{F}: \mathcal{K}\left(\mathbb{R}^{N}\right) \rightsquigarrow \operatorname{Lip}\left(\mathbb{R}^{N}, \mathbb{R}^{N}\right)$. Correspondingly to Definition 2.6, we introduce the solution of a morphological inclusion in the following way:

Definition 3.1. For any given function $\mathcal{F}: \mathcal{K}\left(\mathbb{R}^{N}\right) \rightsquigarrow \operatorname{Lip}\left(\mathbb{R}^{N}, \mathbb{R}^{N}\right)$, a compact-valued $K(\cdot):\left[0, T\left[\rightsquigarrow \mathbb{R}^{N}\right.\right.$ is called solution of the morphological inclusion

$$
\stackrel{\circ}{K}(\cdot) \cap \mathcal{F}(K(\cdot), \cdot) \neq \emptyset
$$

if 1. $K(\cdot):\left[0, T\left[\rightsquigarrow \mathbb{R}^{N}\right.\right.$ is Lipschitz continuous with respect to $d$ and

2. $\mathcal{F}(K(t)) \cap \stackrel{\circ}{K}(t) \neq \emptyset$ for almost every $t$, i.e. some $w \in \mathcal{F}(K(t)) \subset \operatorname{Lip}\left(\mathbb{R}^{N}, \mathbb{R}^{N}\right)$ belongs to $\stackrel{\circ}{K}(t)$ or, equivalently, $\lim _{h \downarrow 0} \frac{1}{h} \cdot d l\left(K(t+h), \vartheta_{w}(h, K(t))\right)=0$, 
3.1. The (well-known) Viability Theorem for differential inclusions. The situation has already been investigated intensively for differential inclusions in $\mathbb{R}^{N}$ (see e.g. $[5,6]$ ). For clarifying the new aspects of morphological inclusions, we now quote the corresponding result from [5, Theorems 3.3.2, 3.3.5] after specifying the required terms.

Definition 3.2 ([5, Definition 2.2.4]). Let $X$ and $Y$ be normed vector spaces. A set-valued map $F: X \rightsquigarrow Y$ is called Marchaud map if it has the following properties:

1. $F$ is nontrivial, i.e. Graph $F \neq \emptyset$,

2. $F$ is upper semicontinuous, i.e. for any $x \in X$, neighborhood $V \supset F(x)$,

3. F has compact convex values,

4. F has linear growth, $\quad$ i.e. $\sup _{y \in F(x)}|y| \leq C(1+|x|)$ for all $x \in X$.

TheOREM 3.3 (Viability theorem for differential inclusions [5, Th. 3.3.2, 3.3.5]). Consider a Marchaud map $F: \mathbb{R}^{N} \rightsquigarrow \mathbb{R}^{N}$ and a nonempty closed subset $V \subset \mathbb{R}^{N}$ with $F(x) \neq \emptyset$ for all $x \in V$.

Then for any $T \in] 0, \infty[$, the following two statements are equivalent:

1. For every point $x_{0} \in V$, there is at least one solution $x(\cdot) \in W^{1,1}\left([0, T], \mathbb{R}^{N}\right)$ of $x^{\prime}(\cdot) \in F(x(\cdot))$ (almost everywhere) with $x(0)=x_{0}$ and $x(t) \in V$ for all $t$.

2. $F(x) \cap T_{V}(x) \neq \emptyset$ for all $x \in V$.

The implication (1.) $\Longrightarrow(2$.) is rather obvious. For proving (2.) $\Longrightarrow(1$.), a standard approach uses an "approximating" sequence $\left(x_{n}(\cdot)\right)_{n \in \mathbb{N}}$ in $W^{1, \infty}\left([0,1], \mathbb{R}^{N}\right)$ such that $\sup _{t} \operatorname{dist}\left(x_{n}(t), V\right) \longrightarrow 0(n \rightarrow \infty)$ and $\left(x_{n}(t), \frac{d}{d t} x_{n}(t)\right)$ is close to Graph $F \subset \mathbb{R}^{N} \times \mathbb{R}^{N}$ for almost every $t$. Then the theorems of Arzela-Ascoli and Alaoglu provide a subsequence $\left(x_{n_{j}}(\cdot)\right)_{j \in \mathbb{N}}$ and limits $x(\cdot) \in C^{0}\left([0,1], \mathbb{R}^{N}\right), w(\cdot) \in L^{\infty}\left([0,1], \mathbb{R}^{N}\right)$ with

$$
x_{n_{j}}(\cdot) \longrightarrow x(\cdot) \text { uniformly, } \quad \frac{d}{d t} x_{n_{j}}(\cdot) \longrightarrow w(\cdot) \text { weakly* in } L^{\infty}\left([0,1], \mathbb{R}^{N}\right) .
$$

Due to the continuous embedding $L^{\infty}\left([0,1], \mathbb{R}^{N}\right) \subset L^{1}\left([0,1], \mathbb{R}^{N}\right)$, we even obtain the convergence $\frac{d}{d t} x_{n_{j}}(\cdot) \longrightarrow w(\cdot)$ weakly in $L^{1}\left([0,1], \mathbb{R}^{N}\right)$. Thus, $w(\cdot)$ is the weak derivative of $x(\cdot)$ and, $x(\cdot)$ is Lipschitz continuous. Finally Mazur's Lemma 3.5 implies

$$
w(t) \in \bigcap_{\varepsilon>0} \overline{\mathrm{co}}\left(\bigcup_{z \in \mathbb{B}_{\varepsilon}(x(t))} F(z)\right)=F(x(t)) \quad \text { for almost every } t .
$$

Considering now morphological inclusions on $\left(\mathcal{K}\left(\mathbb{R}^{N}\right), d\right)$ (instead of differential inclusions), an essential aspect changes: The derivative of a curve is not represented as a function in $L^{1}\left([0,1], \mathbb{R}^{N}\right)$ any longer, but rather as a function $[0,1] \longrightarrow \operatorname{Lip}\left(\mathbb{R}^{N}, \mathbb{R}^{N}\right)$. So the classical theorems of Arzela-Ascoli, Alaoglu and Mazur might have to be replaced by their counterparts concerning functions with their values in a Banach space (instead of $\mathbb{R}^{N}$ ).

\subsection{Tools for functions with values in metric or Banach spaces.}

Before adapting this concept for finite-dimensional differential inclusions to Banachvalued functions, we collect briefly the main tools in this framework. They consist mainly of (particularly weakly sequential) compactness criteria for both Bochnerintegrable functions on a probabilistic space and continuous functions on a compact Hausdorff space. 
First of all, the theorems of Arzela-Ascoli and Mazur do not change significantly. Indeed, we always use the following general versions in this paper:

Proposition 3.4 (Arzela-Ascoli in metric spaces [21]). Let $\left(E_{1}, d_{1}\right),\left(E_{2}, d_{2}\right)$ be precompact metric spaces, i.e. for any $\varepsilon>0$, each set $E_{i}$ $(i=1,2)$ can be covered by finitely many $\varepsilon$-balls with respect to metric $d_{i}$. Moreover, suppose the sequence $\left(f_{n}\right)_{n \in \mathbb{N}}$ of functions $E_{1} \longrightarrow E_{2}$ to be uniformly equicontinuous (i.e. with a common modulus of continuity in $\left.E_{1}\right)$.

Then there exists a sequence $n_{j} \nearrow \infty$ such that $\left(f_{n_{j}}\right)_{j \in \mathbb{N}}$ is Cauchy sequence with respect to uniform convergence. If $\left(E_{2}, d_{2}\right)$ is complete in addition, then $\left(f_{n_{j}}\right)_{j \in \mathbb{N}}$ converges uniformly to a continuous function $E_{1} \longrightarrow E_{2}$.

Proposition 3.5 (Mazur's Lemma, e.g. [36, § V.1, Theorem 2]).

For any weakly converging sequence $\left(x_{n}\right)_{n \in \mathbb{N}}$ in a normed vector space, its weak limit is contained in the closed convex hull of $\left\{x_{n} \mid n \in \mathbb{N}\right\}$.

The so-called Bochner integral extends the familiar concept of integration from realvalued functions to Banach-valued functions on the basis of "simple" functions.

Definition $3.6([15])$. Let $(\Omega, \Sigma, \mu)$ be a finite measure space and $X$ a Banach space. A function $f: \Omega \longrightarrow X$ is called simple if there exist $x_{1}, x_{2} \ldots x_{n} \in X$ and $E_{1}, E_{2} \ldots E_{n} \in \Sigma$ such that $f=\sum_{j=1}^{n} x_{j} \chi_{E_{j}}$ with $\chi_{E_{j}}: \Omega \longrightarrow\{0,1\}$ denoting the characteristic function of $E_{j} \subset \Omega$.

A function $f: \Omega \longrightarrow X$ is called $\mu$-measurable if there exists a sequence $\left(f_{n}\right)_{n \in \mathbb{N}}$ of simple functions $\Omega \longrightarrow X$ with $\left\|f-f_{n}\right\|_{X} \longrightarrow 0 \mu$-almost everywhere for $n \rightarrow \infty$.

A $\mu$-measurable function $f: \Omega \longrightarrow X$ is called Bochner integrable if there exists a sequence $\left(f_{n}\right)_{n \in \mathbb{N}}$ of simple functions $\Omega \longrightarrow X$ such that

$$
\lim _{n \rightarrow \infty} \int_{\Omega}\left\|f-f_{n}\right\|_{X} d \mu=0 .
$$

Then, the Bochner integral of $f$ over $E \in \Sigma$ is defined by $\int_{E} f d \mu:=\lim _{n \rightarrow \infty} \int_{E} f_{n} d \mu$. Let $L^{1}(\mu, X)$ denote the Banach space of Bochner integrable functions $\Omega \longrightarrow X$ equipped with its usual $L^{1}$ norm.

In the nineties, Ülger proved that restricting the values of Bochner integrable functions to a weakly compact subset of $X$ implies the relative weak compactness of these functions in $L^{1}(\mu, X)$. For real-valued Lebesgue integrable functions, this is closely related with Alaoglu's Theorem and a compact embedding.

Proposition 3.7 ([34, Proposition 7]). Let $(\Omega, \Sigma, \mu)$ be a probabilistic space, $X$ an arbitrary Banach space. For any weakly compact subset $W \subset X$, the set

$$
\left\{h \in L^{1}(\mu, X) \mid h(\omega) \in W \text { for } \mu \text {-almost every } \omega \in \Omega\right\}
$$

is relatively weakly compact.

An earlier version of this result is presented in [13] and, [14] considers weak compactness of Bochner integrable functions with values in an arbitrary Banach space under weaker assumptions (see also [8]).

The next proposition of Ülger provides a "weakly pointwise" characterization of weakly convergent sequences in $L^{1}(\mu, X)$. 
Proposition $3.8([34$, Corollary 5$])$. Let $(\Omega, \Sigma, \mu)$ be a probabilistic space and $X$ an arbitrary Banach space as in Proposition 3.7.

Set $W:=\left\{g \in L^{1}(\mu, X)|| g(\omega) \mid \leq 1\right.$ for $\mu$-almost every $\left.\omega \in \Omega\right\}$.

A sequence $\left(g_{n}(\cdot)\right)_{n \in \mathbb{N}}$ in $W \subset L^{1}(\mu, X)$ converges weakly to $g \in L^{1}(\mu, X)$ if and only if for any subsequence $\left(g_{n_{k}}(\cdot)\right)_{k \in \mathbb{N}}$ given, there exists a sequence $\left(h_{k}(\cdot)\right)_{k \in \mathbb{N}}$ with $h_{k} \in \operatorname{co}\left\{g_{n_{k}}, g_{n_{k+1}} \ldots\right\}$ such that for $\mu$-almost every $\omega \in \Omega$,

$$
h_{k}(\omega) \longrightarrow g(\omega) \quad(k \longrightarrow \infty) \quad \text { weakly in } X .
$$

In fact, the classical theorem of Scorza-Dragoni [32] has a counterpart for Banachvalued functions as shown by Ricceri and Villani. A so-called Carathéodory function depends on two arguments and, it is measurable with respect to the first one and continuous with respect to second one. The key point of Scorza-Dragoni is to ensure continuity with respect to both arguments on "almost" the whole domain in the following sense:

Proposition 3.9 ([31, Theorem 1]). Let $S$ be a compact Hausdorff topological space, $\mu$ a Radon measure on $S$ and $X, Y$ metric spaces. Suppose $X$ to be separable. Then every Carathéodory function $g: S \times X \longrightarrow Y$ satisfies the so-called ScorzaDragoni property, i.e. for every $\varepsilon>0$, there exists a closed subset $S_{\varepsilon} \subset S$ with $\mu\left(S \backslash S_{\varepsilon}\right)<\varepsilon$ such that the restriction $\left.f\right|_{S_{\varepsilon} \times X}$ is continuous.

So this proposition can be regarded as a counterpart of well-known Lusin's Theorem (relating measurability to continuity almost everywhere) - but for functions with two arguments.

Last but not least, we quote a result of Kisielewicz characterizing weakly converging sequences of continuous functions on a compact Hausdorff space (like $[0, T] \subset \mathbb{R}$ ).

Proposition 3.10 ([23, Theorem 3]). Let $S$ be a compact Hausdorff space and $X$ an arbitrary Banach space. $C^{0}(S, X)$ denotes the Banach space of continuous functions $S \longrightarrow X$ supplied with the supremum norm $\|\cdot\|_{\infty}$.

A sequence $\left(g_{n}(\cdot)\right)_{n \in \mathbb{N}}$ in $C^{0}(S, X)$ converges weakly to $g \in C^{0}(S, X)$ if and only if

$$
\wedge\left\{\begin{array}{lll}
\sup _{n}\left\|g_{n}\right\|_{\infty}<\infty & \text { and } \\
g_{n}(s) \longrightarrow g(s) & \text { weakly in } X \quad(n \longrightarrow \infty) \quad \text { for every } s \in S .
\end{array}\right.
$$

\subsection{Adapting this concept to morphological inclusions.}

Now $\mathcal{F}: \mathcal{K}\left(\mathbb{R}^{N}\right) \rightsquigarrow \operatorname{Lip}\left(\mathbb{R}^{N}, \mathbb{R}^{N}\right)$ and a constrained set $\mathcal{V} \subset \mathcal{K}\left(\mathbb{R}^{N}\right)$ are given.

Correspondingly to Theorem 3.3 about differential inclusions, we focus on the so-called viability condition demanding from each compact set $K \in \mathcal{V}$ that the value $\mathcal{F}(K)$ and the contingent transition set $T_{\mathcal{V}}(K) \subset \operatorname{Lip}\left(\mathbb{R}^{N}, \mathbb{R}^{N}\right)$ have at least one transition in common. Lacking a concrete counterpart of Aumann integral in the metric space $\left(\mathcal{K}\left(\mathbb{R}^{N}\right), d\right)$, the question of its necessity (for the existence of "in $\mathcal{V}$ viable" solutions) is more complicated than for differential inclusions in $\mathbb{R}^{N}$ and thus, we skip it here deliberately.

The main contribution of this paper is that in combination with appropriate assumptions about $\mathcal{F}(\cdot)$ and $\mathcal{V}$, the viability condition is sufficient. The proof is given in several steps by approximative solutions: 
TheOREM 3.11. Let $\mathcal{F}: \mathcal{K}\left(\mathbb{R}^{N}\right) \rightsquigarrow \operatorname{Lip}\left(\mathbb{R}^{N}, \mathbb{R}^{N}\right)$ be a set-valued map and $\mathcal{V} \subset \mathcal{K}\left(\mathbb{R}^{N}\right)$ a nonempty closed subset satisfying:

1.) all values of $\mathcal{F}$ are nonempty and convex,

2.) $A:=\sup _{M \in \mathcal{K}\left(\mathbb{R}^{N}\right)} \sup _{f \in \mathcal{F}(M)} \operatorname{Lip} f<\infty$,

$B:=\sup _{M \in \mathcal{K}\left(\mathbb{R}^{N}\right)} \sup _{f \in \mathcal{F}(M)}\|f\|_{\infty}<\infty$

3.) the graph of $\mathcal{F}$ is closed (w.r.t. locally uniform convergence in $\operatorname{Lip}\left(\mathbb{R}^{N}, \mathbb{R}^{N}\right)$ ),

4.) $T_{\mathcal{V}}\left(K_{0}\right) \cap \mathcal{F}\left(K_{0}\right) \neq \emptyset \quad$ for all $K_{0} \in \mathcal{V}$.

Then for every initial compact set $K_{0} \in \mathcal{V}$, there exists a compact-valued Lipschitz continuous solution $K(\cdot):[0,1] \rightsquigarrow \mathbb{R}^{N}$ of $\stackrel{\circ}{K}(\cdot) \cap \mathcal{F}(K(\cdot)) \neq \emptyset$ with $K(0)=K_{0}$ and $K(t) \in \mathcal{V}$ for all $t \in[0,1]$.

LEMMA 3.12 (Constructing approximative solutions). Choose any $\varepsilon>0$. Under the assumptions of Viability Theorem 3.11, there exist a B-Lipschitz continuous function $K_{\varepsilon}(\cdot):[0,1] \longrightarrow \mathcal{K}\left(\mathbb{R}^{N}\right)$ and a piecewise constant function $f_{\varepsilon}(\cdot)$ : $\left[0,1\left[\longrightarrow \operatorname{Lip}\left(\mathbb{R}^{N}, \mathbb{R}^{N}\right)\right.\right.$ satisfying with $R_{\varepsilon}:=\varepsilon e^{A}$

a) $K_{\varepsilon}(0)=K_{0}$

b) $\operatorname{dist}\left(K_{\varepsilon}(t), \mathcal{V}\right) \leq R_{\varepsilon} \quad$ for all $t \in[0,1]$,

c) $f_{\varepsilon}(t) \in \stackrel{\circ}{K}_{\varepsilon}(t) \cap \mathcal{F}\left(\mathbb{B}_{R_{\varepsilon}}\left(K_{\varepsilon}(t)\right)\right) \neq \emptyset$ for all $t \in[0,1[$.

Proof. follows the same track as [2, Lemma 1.6.5] and uses Zorn's Lemma: For $\varepsilon>0$ fixed, let $\mathcal{A}_{\varepsilon}\left(K_{0}\right)$ denote the set of all tuples $\left(\tau_{K}, K(\cdot), f(\cdot)\right)$ consisting of some $\tau_{K} \in[0,1]$, a $B$-Lipschitz continuous function $K(\cdot):\left[0, \tau_{K}\right] \longrightarrow\left(\mathcal{K}\left(\mathbb{R}^{N}\right), d l\right)$ and some piecewise constant function $f(\cdot):\left[0,1\left[\longrightarrow \operatorname{Lip}\left(\mathbb{R}^{N}, \mathbb{R}^{N}\right)\right.\right.$ such that

a) $\quad K(0)=K_{0}$,

b') 1.) $\operatorname{dist}\left(K\left(\tau_{K}\right), \mathcal{V}\right) \leq r_{\varepsilon}\left(\tau_{K}\right)$ with $r_{\varepsilon}(t):=\varepsilon e^{A t} t$,

2.) $\operatorname{dist}(K(t), \quad \mathcal{V}) \leq R_{\varepsilon} \quad$ for all $t \in\left[0, \tau_{K}\right]$,

c) $\quad f(t) \in \stackrel{\circ}{K}(t) \cap \mathcal{F}\left(\mathbb{B}_{R_{\varepsilon}}(K(t))\right) \neq \emptyset \quad$ for all $t \in\left[0, \tau_{K}[\right.$.

Obviously, $\mathcal{A}_{\varepsilon}\left(K_{0}\right)$ is not empty since it contains $\left(0, K(\cdot) \equiv K_{0}, f(\cdot) \equiv f_{0}\right)$ with arbitrary $f_{0} \in \operatorname{Lip}\left(\mathbb{R}^{N}, \mathbb{R}^{N}\right)$. Moreover, an order relation $\preceq$ on $\mathcal{A}_{\varepsilon}\left(K_{0}\right)$ is specified by $\left(\tau_{K}, K(\cdot), f(\cdot)\right) \preceq\left(\tau_{M}, M(\cdot), g(\cdot)\right) \quad: \Longleftrightarrow \tau_{K} \leq \tau_{M},\left.M\right|_{\left[0, \tau_{K}\right]}=K,\left.g\right|_{\left[0, \tau_{K}[\right.}=f$.

So Zorn's Lemma provides a maximal element $\left(\tau, K_{\varepsilon}(\cdot), f_{\varepsilon}(\cdot)\right) \in \mathcal{A}_{\varepsilon}\left(K_{0}\right)$.

As all considered functions with values in $\mathcal{K}\left(\mathbb{R}^{N}\right)$ have been supposed to be $B$ Lipschitz continuous, $K_{\varepsilon}(\cdot)$ is well-defined on the closed interval $[0, \tau] \subset[0,1]$.

Assuming $\tau<1$ for a moment, we obtain a contradiction if $K_{\varepsilon}(\cdot), f_{\varepsilon}(\cdot)$ can be extended to a larger interval $[0, \tau+\delta] \subset[0,1](\delta>0)$ preserving conditions (b'), (c). Since closed bounded balls of $\left(\mathcal{K}\left(\mathbb{R}^{N}\right), d l\right)$ are compact, the closed set $\mathcal{V}$ contains an element $Z \in \mathcal{K}\left(\mathbb{R}^{N}\right)$ with $d\left(K_{\varepsilon}(\tau), Z\right)=\operatorname{dist}\left(K_{\varepsilon}(\tau), \mathcal{V}\right) \leq r_{\varepsilon}(\tau)$ and, assumption (4.) of Viability Theorem 3.11 provides an element

$$
g \in T_{\mathcal{V}}(Z) \cap \mathcal{F}(Z) \subset \operatorname{Lip}\left(\mathbb{R}^{N}, \mathbb{R}^{N}\right) .
$$

Due to Definition 2.9 of the contingent transition set $T_{\mathcal{V}}(Z)$, there is a sequence $h_{m} \downarrow 0$ in $] 0,1-\tau\left[\operatorname{such}\right.$ that $\operatorname{dist}\left(\vartheta_{g}\left(h_{m}, Z\right), \mathcal{V}\right) \leq \varepsilon h_{m}$ for all $m \in \mathbb{N}$. Now set

$$
K_{\varepsilon}(t):=\vartheta_{g}\left(t-\tau, K_{\varepsilon}(\tau)\right), \quad f_{\varepsilon}(t):=g \quad \text { for each } t \in\left[\tau, \tau+h_{1}\right] .
$$


Obviously, Lemma 2.4 implies $g \in \stackrel{\circ}{K}_{\varepsilon}(t)$ for all $t \in\left[\tau, \tau+h_{1}[\right.$. Moreover, it leads to

$$
\begin{aligned}
d l\left(K_{\varepsilon}(t), Z\right) & \leq d\left(\vartheta_{g}\left(t-\tau, K_{\varepsilon}(\tau)\right), K_{\varepsilon}(\tau)\right) & +d\left(K_{\varepsilon}(\tau), Z\right) \\
& \leq B \cdot(t-\tau) & +\varepsilon e^{A \tau} \tau \quad \leq R_{\varepsilon}
\end{aligned}
$$

for every $t \in\left[\tau, \tau+\delta\left[\right.\right.$ with $\delta:=\min \left\{h_{1}, \varepsilon e^{A} \frac{1-\tau}{1+B}\right\}$, i.e. conditions (b')(2.) and (c) hold in the interval $[\tau, \tau+\delta]$. For any index $m \in \mathbb{N}$ with $h_{m}<\delta$,

$$
\begin{aligned}
\operatorname{dist}\left(K_{\varepsilon}\left(\tau+h_{m}\right), \mathcal{V}\right) & \leq d\left(\vartheta_{g}\left(h_{m}, K_{\varepsilon}(\tau)\right), \vartheta_{g}\left(h_{m}, Z\right)\right)+\operatorname{dist}\left(\vartheta_{g}\left(h_{m}, Z\right), \mathcal{V}\right) \\
& \leq d\left(K_{\varepsilon}(\tau), Z\right) \cdot e^{A h_{m}}+\varepsilon \cdot h_{m} \\
& \leq \varepsilon e^{A \tau} \tau \cdot e^{A h_{m}} \quad+\varepsilon \cdot h_{m} \leq r_{\varepsilon}\left(\tau+h_{m}\right),
\end{aligned}
$$

i.e. condition (b')(2.) is also satisfied at time $t=\tau+h_{m}$ with any large $m \in \mathbb{N}$.

So $\left.K_{\varepsilon}(\cdot)\right|_{\left[0, \tau+h_{m}\right]}$ and $\left.f_{\varepsilon}(\cdot)\right|_{\left[0, \tau+h_{m}[\right.}$ provide the wanted contradiction and thus, $\tau=1$.

Using the abbreviation $\widetilde{K}_{j}:=\mathbb{B}_{j+B}\left(K_{0}\right) \stackrel{\text { Def. }}{=}\left\{x \in \mathbb{R}^{N} \mid \operatorname{dist}\left(x, K_{0}\right) \leq j+B\right\}(j \in \mathbb{N})$ for (arbitrarily large) compact neighborhoods of the initial set $K_{0}$, we obtain

LEMma 3.13 (Selecting an approximative subsequence). Under the assumptions of Viability Theorem 3.11, there are sequences $K_{n}(\cdot):[0,1] \longrightarrow \mathcal{K}\left(\mathbb{R}^{N}\right)$, $f_{n}(\cdot):\left[0,1\left[\longrightarrow \operatorname{Lip}\left(\mathbb{R}^{N}, \mathbb{R}^{N}\right)(n \in \mathbb{N})\right.\right.$ and functions $K(\cdot):[0,1] \longrightarrow \mathcal{K}\left(\mathbb{R}^{N}\right)$, $f(\cdot):\left[0,1\left[\longrightarrow \operatorname{Lip}\left(\mathbb{R}^{N}, \mathbb{R}^{N}\right)\right.\right.$ such that for every $j, n \in \mathbb{N}$,

a) $K_{0}=K_{n}(0)=K(0)$,

b) $K(\cdot)$ and $K_{n}(\cdot)$ are B-Lipschitz continuous w.r.t. $d$,

c) $f_{n}(\cdot)$ is piecewise constant, $\sup _{t \in[0,1[} \operatorname{Lip} f_{n}(t) \leq A<\infty, \quad \sup _{t \in[0,1[}\left\|f_{n}(t)\right\|_{\infty} \leq B<\infty$,

d) $\operatorname{dist}\left(K_{n}(t), \mathcal{V}\right) \leq \frac{1}{n}$

for all $t \leq 1$,

e) $f_{n}(t) \in \stackrel{\circ}{K}_{n}(t) \cap \mathcal{F}\left(\mathbb{B}_{1 / n}\left(K_{n}(t)\right)\right) \neq \emptyset$

for all $t<1$,

f) $d\left(K_{m}(\cdot), K(\cdot)\right) \longrightarrow 0 \quad$ uniformly in $[0,1] \quad$ for $\quad m \longrightarrow \infty$,

g) $\left.\left.f_{m}(\cdot)\right|_{\widetilde{K}_{j}} \longrightarrow f(\cdot)\right|_{\widetilde{K}_{j}} \quad$ weakly in $L^{1}\left([0,1], C^{0}\left(\widetilde{K}_{j}, \mathbb{R}^{N}\right)\right) \quad$ for $\quad m \longrightarrow \infty$,

h) $\operatorname{Lip} f(t)(\cdot) \leq A, \quad\|f(t)(\cdot)\|_{\infty} \leq B \quad$ for a.e. $t<1$.

Proof. is based on the approximative solutions of Lemma 3.12, of course. Indeed, for each index $n \in \mathbb{N}$, Lemma 3.12 provides $K_{n}(\cdot):[0,1] \longrightarrow \mathcal{K}\left(\mathbb{R}^{N}\right)$ and $f_{n}(\cdot):\left[0,1\left[\longrightarrow \operatorname{Lip}\left(\mathbb{R}^{N}, \mathbb{R}^{N}\right)\right.\right.$ corresponding to $\varepsilon:=\frac{1}{n} e^{-A}$. Obviously, they satisfy the properties (a) - (e) claimed here.

In particular, these features stay correct whenever we consider subsequences instead and again abbreviate them as $\left(K_{n}(\cdot)\right)_{n \in \mathbb{N}},\left(f_{n}(\cdot)\right)_{n \in \mathbb{N}}$ respectively.

For property $(f)$ about uniform convergence of $\left(K_{n}(\cdot)\right)$ w.r.t. $d l$ :

The $B$-Lipschitz continuity of each $K_{n}(\cdot)$ has two important consequences, i.e.

1. all $K_{n}(\cdot):[0,1] \longrightarrow\left(\mathcal{K}\left(\mathbb{R}^{N}\right), d\right)(n \in \mathbb{N})$ are equi-continuous and

2. $\bigcup_{\substack{n \in \mathbb{N} \\ t \in[0,1]}}\left\{K_{n}(t)\right\}$ is contained in the compact subset $\mathbb{B}_{B}\left(K_{0}\right)$ of $\left(\mathcal{K}\left(\mathbb{R}^{N}\right), d\right)$.

So, Proposition 3.4 of Arzela-Ascoli provides a subsequence (again denoted by) $\left(K_{n}(\cdot)\right)_{n}$ converging uniformly to a continuous function $K(\cdot):[0,1] \longrightarrow\left(\mathcal{K}\left(\mathbb{R}^{N}\right), d\right)$. In particular, $K(\cdot)$ is also $B$-Lipschitz continuous with $K(0)=K_{0}$, i.e. properties (a) - (f) are fulfilled completely. 
For property $(g)$ about weak convergence of $\left.f_{n}(\cdot)\right|_{\widetilde{K}}$ with fixed compact $\widetilde{K} \subset \mathbb{R}^{N}$ : We cannot follow the same track as for differential inclusions any longer. Indeed, the functions $f_{n}(\cdot)$ of shape transitions have their values in $\operatorname{Lip}\left(\mathbb{R}^{N}, \mathbb{R}^{N}\right)$ which cannot be regarded as a dual space in an obvious way. So Alaoglu's Theorem (stating that closed balls of dual Banach spaces are weakly* compact) cannot be applied similarly to differential inclusions.

Alternatively, we restrict our considerations to a compact neighborhood $\widetilde{K}$ of $\bigcup_{\substack{n \in \mathbb{N} \\ t \in 0,1]}} K_{n}(t) \subset \mathbb{R}^{N}$ and use a sufficient condition on relatively weakly compact sets in $L^{1}\left([0,1], C^{0}\left(\widetilde{K}, \mathbb{R}^{N}\right)\right)$. Here $C^{0}\left(\widetilde{K}, \mathbb{R}^{N}\right)$ (supplied with the supremum norm $\|\cdot\|_{\infty}$ ) denotes the Banach space of all continuous functions $\widetilde{K} \longrightarrow \mathbb{R}^{N}$.

In fact, the set $\left\{f_{n}(t) \mid n \in \mathbb{N}, t \in[0,1]\right\} \subset C^{0}\left(\mathbb{R}^{N}, \mathbb{R}^{N}\right)$ is uniformly bounded by $B$ and equi-continuous (due to property (c)). So according to Proposition 3.4 of Arzela-Ascoli, the set of their restrictions to $\widetilde{K}$

$$
W:=\left\{\left.f_{n}(t)\right|_{\widetilde{K}} \mid n \in \mathbb{N}, t \in[0,1]\right\} \subset C^{0}\left(\widetilde{K}, \mathbb{R}^{N}\right)
$$

is relatively compact with respect to $\|\cdot\|_{\infty}$. Thus, $\left\{\left.f_{n}(\cdot)\right|_{\widetilde{K}} \mid n \in \mathbb{N}\right\}$ is relatively weakly compact in $L^{1}\left([0,1], C^{0}\left(\widetilde{K}, \mathbb{R}^{N}\right)\right)$ according to Proposition 3.7 and, we obtain a subsequence (again denoted by) $\left(f_{n}(\cdot)\right)_{n \in \mathbb{N}}$ and some $g(\cdot) \in L^{1}\left([0,1], C^{0}\left(\widetilde{K}, \mathbb{R}^{N}\right)\right)$ with $\left.\quad f_{n}(\cdot)\right|_{\widetilde{K}} \longrightarrow g(\cdot) \quad$ weakly in $L^{1}\left([0,1], C^{0}\left(\widetilde{K}, \mathbb{R}^{N}\right)\right)$. Obviously, both the subsequence and $g(\cdot)$ depend on $\widetilde{K}$, however.

For property $(g)$ about $\left.f_{n}(\cdot)\right|_{\widetilde{K}_{j}}$ with every compact $\widetilde{K}_{j} \subset \mathbb{R}^{N}(j \in \mathbb{N})$ :

Now this construction of subsequences is applied to the compact subsets $\widetilde{K}_{j} \stackrel{\text { Def. }}{=}$ $\mathbb{B}_{j+B}\left(K_{0}\right)$ of $\mathbb{R}^{N}$ for $j=1,2,3 \ldots$ successively. By means of Cantor's diagonal construction, we obtain a subsequence (again denoted by) $\left(f_{n}(\cdot)\right)_{n \in \mathbb{N}}$ and a function $g_{j}(\cdot) \in L^{1}\left([0,1], C^{0}\left(\widetilde{K}_{j}, \mathbb{R}^{N}\right)\right)$ (for each $j \in \mathbb{N}$ ) such that for all $j \in \mathbb{N}$,

$$
\left.f_{n}(\cdot)\right|_{\widetilde{K}_{j}} \longrightarrow g_{j}(\cdot) \quad \text { weakly in } L^{1}\left([0,1], C^{0}\left(\widetilde{K}_{j}, \mathbb{R}^{N}\right)\right) .
$$

As restrictions to $\widetilde{K}_{j}$ of one and the same subsequence $\left(f_{n}(\cdot)\right)_{n \in \mathbb{N}}$ converge weakly for each $j \in \mathbb{N}$, the inclusion $\widetilde{K}_{j} \subset \widetilde{K}_{j+1}$ implies for any indices $j<k$

$$
g_{j}(t)(\cdot)=\left.g_{k}(t)(\cdot)\right|_{\widetilde{K}_{j}} \in C^{0}\left(\widetilde{K}_{j}, \mathbb{R}^{N}\right) \quad \text { for almost every } t \in[0,1]
$$

and, so $\left(g_{j}(\cdot)\right)_{j \in \mathbb{N}}$ induces a single function $f:\left[0,1\left[\longrightarrow C^{0}\left(\mathbb{R}^{N}, \mathbb{R}^{N}\right)\right.\right.$ defined as

$$
f(t)(x):=g_{j}(t)(x) \quad \text { for } x \in \widetilde{K}_{j} \text { and almost every } t \in[0,1[.
$$

For property $(h)$ about Lipschitz continuity and bounds of limit function $f(\cdot)$ :

Finally, we verify $f(t) \in \operatorname{Lip}\left(\mathbb{R}^{N}, \mathbb{R}^{N}\right), \quad \operatorname{Lip} f(t) \leq A$ and $\|f(t)\|_{\infty} \leq B$ for almost every $t \in[0,1$. Indeed, as in the case of differential inclusions (§ 3.1), Mazur's Lemma 3.5 ensures here for each $j \in \mathbb{N}$ (fixed)

$$
\left.f(\cdot)\right|_{\widetilde{K}_{j}} \in \bigcap_{n \in \mathbb{N}} \overline{c o}\left\{\left.f_{n}(\cdot)\right|_{\widetilde{K}_{j}},\left.f_{n+1}(\cdot)\right|_{\widetilde{K}_{j}} \ldots\right\} \quad \text { in } L^{1}\left([0,1], C^{0}\left(\widetilde{K}_{j}, \mathbb{R}^{N}\right)\right) \text {. }
$$

Thus, $\left.f(\cdot)\right|_{\widetilde{K}_{j}}$ can be approximated by convex combinations of $\left\{\left.f_{1}(\cdot)\right|_{\widetilde{K}_{j}},\left.f_{2}(\cdot)\right|_{\widetilde{K}_{j}} \ldots\right\}$ with respect to the $L^{1}$ norm. A further subsequence (of these convex combinations) converges to $\left.f(\cdot)\right|_{\widetilde{K}_{j}}$ almost everywhere in $[0,1]$. So, for almost every $t \in[0,1]$, $\left.f(t)\right|_{\widetilde{K}_{j}}$ belongs to the same compact convex subset of $\left(C^{0}\left(\widetilde{K}_{j}, \mathbb{R}^{N}\right),\|\cdot\|_{\infty}\right)$ as $\left.f_{1}(t)\right|_{\widetilde{K}_{j}},\left.f_{2}(t)\right|_{\widetilde{K}_{j}} \ldots$, namely $\left\{w \in \operatorname{Lip}\left(\widetilde{K}_{j}, \mathbb{R}^{N}\right) \mid \operatorname{Lip} w \leq A,\|w\|_{\infty} \leq B\right\}$. 
LEmma 3.14 (The limit function is a solution). Under the assumptions of Viability Theorem 3.11, consider both $K_{n}(\cdot), K(\cdot):[0,1] \longrightarrow \mathcal{K}\left(\mathbb{R}^{N}\right) \quad(n \in \mathbb{N})$ and $f_{n}(\cdot), f(\cdot):\left[0,1\left[\longrightarrow \operatorname{Lip}\left(\mathbb{R}^{N}, \mathbb{R}^{N}\right)\right.\right.$ specified in Lemma 3.13.

Then $K(\cdot)$ is a solution of the morphological inclusion $\stackrel{\circ}{K}(\cdot) \cap \mathcal{F}(K(\cdot)) \neq \emptyset$ with $K(0)=K_{0}$ and $K(t) \in \mathcal{V}$ for all $t \in[0,1]$.

Proof. $K(t) \in \mathcal{V}$ for all $t \in[0,1]$ results directly from properties (d), (f) of Lemma 3.13 because $\mathcal{V}$ is assumed to be a closed subset of $\left(\mathcal{K}\left(\mathbb{R}^{N}\right), d\right)$.

So $f(t) \in \stackrel{\circ}{K}(\cdot) \cap \mathcal{F}(K(\cdot))$ is still to prove for Lebesgue-almost every $t \in[0,1]$.

The Carathéodory property of each $f_{n}(\cdot)$ and, every $K_{n}(\cdot)$ is reachable set:

As each $f_{n}:\left[0,1\left[\longrightarrow \operatorname{Lip}\left(\mathbb{R}^{N}, \mathbb{R}^{N}\right)\right.\right.$ from Lemma 3.13 is piecewise constant, it can be regarded as a measurable/Lipschitz function $\left[0,1\left[\times \mathbb{R}^{N} \longrightarrow \mathbb{R}^{N},(t, x) \longmapsto f_{n}(t)(x)\right.\right.$ in the sense of [7, Definition 9.5.1], i.e.

$f_{n}(\cdot)(x): \quad\left[0,1\left[\longrightarrow \mathbb{R}^{N} \quad\right.\right.$ is Lebesgue measurable for every $x \in \mathbb{R}^{N} \quad$ and $f_{n}(t)(\cdot): \quad \mathbb{R}^{N} \longrightarrow \mathbb{R}^{N}$ is $A$-Lipschitz continuous for every $t \in[0,1[$.

In addition, $\left\|f_{n}(t)(\cdot)\right\|_{\infty} \leq B$ for every $t \in[0,1[, n \in \mathbb{N}$.

Moreover, each compact set $K_{n}(t) \subset \mathbb{R}^{N}$ coincides with the reachable set

$\vartheta_{f_{n}}\left(t, K_{0}\right) \stackrel{\text { Def. }}{=}\left\{x(t) \mid \exists x \in W^{1,1}\left([0, t], \mathbb{R}^{N}\right): x^{\prime}(s)=f_{n}(s)(x(s))\right.$ for a.e. $\left.s, x(0) \in K_{0}\right\}$

of the initial set $K_{0}$ and the function $f_{n}(\cdot)(\cdot):\left[0,1\left[\times \mathbb{R}^{N} \longrightarrow \mathbb{R}^{N}\right.\right.$. Indeed, consider a subinterval $\left[s_{1}, s_{2}\left[\subset\left[0,1\left[\right.\right.\right.\right.$ in which $f_{n}(\cdot)$ is constant, i.e. $\left.f_{n}(\cdot)\right|_{\left[s_{1}, s_{2}[\right.} \equiv g \in$ $\operatorname{Lip}\left(\mathbb{R}^{N}, \mathbb{R}^{N}\right)$, and assume $K_{\varepsilon}\left(s_{1}\right)=\vartheta_{f_{n}}\left(s_{1}, K_{0}\right)$ (with subsequent induction in mind). Then both $K_{\varepsilon}(\cdot)$ and the reachable set $\vartheta_{f_{n}}\left(\cdot, K_{0}\right)$ satisfy the morphological equation $\stackrel{\circ}{Q}(\cdot) \ni g$ in $\left[s_{1}, s_{2}[\right.$ and are $B$-Lipschitz continuous. So according to Theorem 2.7, $K_{\varepsilon}(\cdot) \equiv \vartheta_{f_{n}}\left(\cdot, K_{0}\right)$ in $\left[s_{1}, s_{2}\right]$. By means of induction, we conclude $K_{\varepsilon}(\cdot) \equiv \vartheta_{f_{n}}\left(\cdot, K_{0}\right)$ in $[0,1]$.

For characterizing $K(t)$ as reachable set of $f(\cdot): K(t) \subset \vartheta_{f}\left(t, K_{0}\right)$ for every $t$. Indeed, Lemma 3.13 (f) implies the characterization as limit with respect to $d$ (or, equivalently for compact sets here, in the sense of Painlevé-Kuratowski)

$$
K(t)=\operatorname{Lim}_{n \rightarrow \infty} K_{n}(t)=\operatorname{Lim}_{n \rightarrow \infty} \vartheta_{f_{n}}\left(t, K_{0}\right) .
$$

For every $x \in K(t)$, there is a sequence $\left(x_{n}(\cdot)\right)_{n \in \mathbb{N}}$ of functions in $W^{1,1}\left([0, t], \mathbb{R}^{N}\right)$ satisfying

$$
\left\{\begin{array}{lll}
x_{n}^{\prime}(s)=f_{n}(s)\left(x_{n}(s)\right) & \text { for a.e. } s \in[0, t], \\
x_{n}(0) \in K_{0}, & & \\
x_{n}(s) \in \vartheta_{f_{n}}\left(s, K_{0}\right) \subset \subset \mathbb{B}_{1+B}\left(K_{0}\right) \stackrel{\text { Def. }}{=} \widetilde{K}_{1} & \text { for each } s \in[0, t], \\
x_{n}(t) \longrightarrow x & \text { for } \quad n \longrightarrow \infty .
\end{array}\right.
$$

Seizing the notions of [5, Convergence Theorem 2.4.4], the theorems of Arzela-Ascoli and Alaoglu provide a subsequence $\left(x_{n_{j}}(\cdot)\right)_{j \in \mathbb{N}}$ and functions $x(\cdot) \in C^{0}\left([0, t], \mathbb{R}^{N}\right)$, $v(\cdot) \in L^{1}\left([0, t], \mathbb{R}^{N}\right)$ such that

$x_{n_{j}}(\cdot) \longrightarrow x(\cdot) \quad$ uniformly in $[0, t], \quad x_{n_{j}}^{\prime}(\cdot) \longrightarrow v(\cdot) \quad$ weakly in $L^{1}\left([0, t], \mathbb{R}^{N}\right)$ implying the absolute continuity of $x(\cdot)$ with $x^{\prime}(\cdot)=v(\cdot)$.

For verifying $x^{\prime}(\cdot)=f(\cdot)(x(\cdot))$ (a.e.), we now prove $f_{n_{j}}(\cdot)\left(x_{n_{j}}(\cdot)\right) \longrightarrow f(\cdot)(x(\cdot))$ weakly in $L^{1}\left([0, t], \mathbb{R}^{N}\right)$ for $j \longrightarrow \infty$. For any $g \in L^{\infty}\left([0, t], \mathbb{R}^{N}\right) \cong\left(L^{1}\left([0, t], \mathbb{R}^{N}\right)\right)^{*}$, 
the $A$-Lipschitz continuity of each $f_{n_{j}}(s)$ implies

$$
\begin{aligned}
\int_{0}^{t} g(s)^{T} f_{n_{j}}(s)\left(x_{n_{j}}(s)\right) d s & \in \int_{0}^{t} g(s)^{T} f_{n_{j}}(s)(x(s)) d s+c\left\|x(\cdot)-x_{n_{j}}(\cdot)\right\|_{\infty} \cdot \mathbb{B}_{1} \\
& \stackrel{j \rightarrow \infty}{\longrightarrow} \int_{0}^{t} g(s)^{T} f(s)(x(s)) \quad d s
\end{aligned}
$$

since $L^{1}\left([0,1], C^{0}\left(\widetilde{K}_{1}, \mathbb{R}^{N}\right)\right) \longrightarrow \mathbb{R}, h \longmapsto \int_{0}^{t} g(s)^{T} h(s)(x(s)) d s$ is continuous and linear. Thus, $x=x(t) \in \vartheta_{f}\left(t, K_{0}\right)$.

For characterizing $K(t)$ as reachable set of $f(\cdot): \vartheta_{f}\left(t, K_{0}\right) \subset K(t)$ for every $t$. The next step is to verify that the tube $K(\cdot):[0,1] \rightsquigarrow \mathbb{R}^{N}$ is invariant under $f$, i.e. for every initial point $x \in K(t)$ (with $t \in\left[0,1[)\right.$, the solution $x(\cdot) \in W^{1,1}\left([t, 1], \mathbb{R}^{N}\right.$ ) of $x^{\prime}(\cdot)=f(\cdot)(x(\cdot))$ (a.e.) with $x(t)=x$ satisfies $x(\tau) \in K(\tau)$ for any $\tau \in[t, 1]$. Due to $K(0)=K_{0}$, this property implies $\vartheta_{f}\left(t, K_{0}\right) \subset K(t)$ for every $t \in[0,1]$.

Indeed, existence and uniqueness of this solution $x(\cdot)$ result from (generalized) Filippov's Theorem (e.g. [35]) since $[0,1] \times \mathbb{R}^{N},(s, y) \longmapsto f(s)(y)$ is measurable/Lipschitz (in the sense of [7, Definition 9.5.1]). Each $x \in K(t)=\operatorname{Lim}_{n \rightarrow \infty} K_{n}(t)$ is limit of a sequence $\left(x_{n}\right)_{n \in \mathbb{N}}$ with $x_{n} \in K_{n}(t)$ and there exist corresponding solutions $x_{n}(\cdot) \in W^{1,1}\left([t, 1], \mathbb{R}^{N}\right)(n \in \mathbb{N})$ of $x_{n}^{\prime}(\cdot)=f_{n}(\cdot)\left(x_{n}(\cdot)\right)$ (a.e.) with $x_{n}(t)=x_{n}$. For the same reasons as before, we obtain a subsequence $\left(x_{n_{j}}(\cdot)\right)_{j \in \mathbb{N}}$ and a limit function $y(\cdot) \in W^{1,1}\left([t, 1], \mathbb{R}^{N}\right)$ satisfying

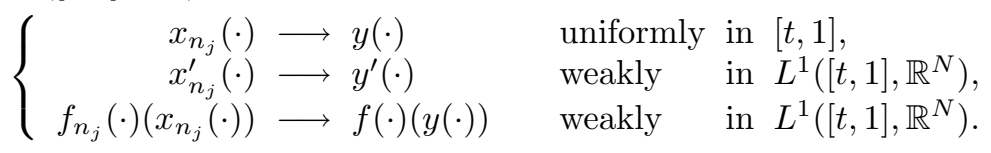

So $y(\cdot)$ is identical to the uniquely determined solution $x(\cdot)$ of $x^{\prime}(\cdot)=f(\cdot)(x(\cdot))$ (a.e.) with $x(t)=x$, i.e. the limit $y(\cdot)$ does not depend on the selection of the subsequence $\left(x_{n_{j}}(\cdot)\right)_{j \in \mathbb{N}}$. This implies indirectly that even the whole sequence $\left(x_{n}(\cdot)\right)_{n \in \mathbb{N}}$ converges to $x(\cdot)$ uniformly and all its derivatives tend weakly to $x^{\prime}(\cdot)$. In particular, $x(\tau)=\lim _{n \rightarrow \infty} x_{n}(\tau) \in \operatorname{Lim}_{n \rightarrow \infty} K_{n}(\tau)=K(\tau)$ for every $\tau \in[t, 1]$.

Thus, $\quad K(t)=\vartheta_{f}\left(t, K_{0}\right) \quad$ for every $t \in[0,1]$.

$K(t)$ as reachable set and Scorza-Dragoni ensure solution property at a.e. time, i.e. describing $K(t)$ as reachable set of $f(\cdot)(\cdot):\left[0,1\left[\times \mathbb{R}^{N} \longrightarrow \mathbb{R}^{N}\right.\right.$ implies that $f(t) \in \operatorname{Lip}\left(\mathbb{R}^{N}, \mathbb{R}^{N}\right)$ belongs to the shape mutation $\stackrel{\circ}{K}(t)$ for a.e. $t \in[0,1]$ :

$$
\lim _{h \downarrow 0} \frac{1}{h} \cdot d l\left(\vartheta_{f(t)}(h, K(t)), K(t+h)\right)=0 .
$$

Indeed, $f(\cdot)(\cdot):\left[0,1\left[\times \mathbb{R}^{N} \longrightarrow \mathbb{R}^{N}\right.\right.$ is measurable/Lipschitz and thus, Proposition 3.9 ensures the following (slightly modified) Scorza-Dragoni property:

For any $\varepsilon>0$, there exists a closed subset $J_{\varepsilon} \subset[0,1-\varepsilon]$ with $\mathcal{L}^{1}\left([0,1] \backslash J_{\varepsilon}\right)<2 \varepsilon$ such that the restriction of $f(\cdot)(\cdot)$ to $J_{\varepsilon} \times \mathbb{R}^{N}$ is continuous. Seizing an idea in the proof of [20, Lemma 2.6], let $\widetilde{J}_{\varepsilon}$ be the subset of all density points of $J_{\varepsilon}$ that are also Lebesgue points of the characteristic function $\chi_{[0,1] \backslash J_{\varepsilon}}(\cdot)$. Then, $\mathcal{L}^{1}\left(\widetilde{J}_{\varepsilon}\right)=\mathcal{L}^{1}\left(J_{\varepsilon}\right)>1-2 \varepsilon$, because Lebesgue points of each $\mathcal{L}^{1}$ function always have full Lebesgue measure [37, Theorem 1.3.8] and so, in particular, density points of any measurable set also have full Lebesgue measure.

For any $t \in \widetilde{J}_{\varepsilon}, x \in K(t)$, there exist unique solutions $x(\cdot), y(\cdot) \in \operatorname{Lip}\left([t, 1], \mathbb{R}^{N}\right)$ of

$$
x^{\prime}(\cdot)=f(\cdot)(x(\cdot)), \quad y^{\prime}(\cdot)=f(t)(y(\cdot)) \quad \text { almost everywhere in }[t, 1],
$$

respectively, with $x(t)=x=y(t)$. Then, we obtain for every $\tau \in] t, 1]$ 


$$
\begin{aligned}
& |x(\tau)-y(\tau)| \\
& =\quad\left|\int_{t}^{\tau}(f(s)(x(s))-f(t)(y(s))) d s\right| \\
& \leq \quad\left|\int_{[t, \tau] \cap J_{\varepsilon}}(f(s)(x(s))-f(t)(y(s))) d s\right|+2 B \cdot \mathcal{L}^{1}\left([t, \tau] \backslash J_{\varepsilon}\right) \\
& \leq \quad \int_{[t, \tau] \cap J_{\varepsilon}}|f(s)(x(s))-f(t)(x(s))| d s+2 B \cdot \mathcal{L}^{1}\left([t, \tau] \backslash J_{\varepsilon}\right) \\
& \quad+\quad \int_{[t, \tau] \cap J_{\varepsilon}} A \cdot|x(s)-y(s)| d s .
\end{aligned}
$$

For $\delta>0$ arbitrarily small and each $t \in \widetilde{J}_{\varepsilon}$, the construction of $J_{\varepsilon}$ and $\widetilde{J}_{\varepsilon}$ provides some $T \in] t, 1]$ satisfying

$$
\left\{\begin{array}{cll}
\sup _{s \in[t, T] \cap J_{\varepsilon}} & \sup _{z: \operatorname{dist}\left(z, K_{0}\right) \leq B} & |f(s)(z)-f(t)(z)|<\delta \\
& \sup _{s \in[t, T]} & \frac{1}{|s-t|} \cdot \mathcal{L}^{1}\left([t, s] \backslash J_{\varepsilon}\right)<\delta
\end{array}\right.
$$

thus, $|x(\tau)-y(\tau)| \leq A \int_{[t, \tau]}|x(s)-y(s)| d s+\delta(1+2 B)(\tau-t)$ for any $\left.\left.\tau \in\right] t, T\right]$. Gronwall's Lemma implies $|x(\tau)-y(\tau)| \leq \delta(1+2 B) e^{A \cdot(\tau-t)}(\tau-t)$ for any $\tau$. As $x \in K(t)$ is chosen arbitrarily and $T$ does not depend on $x$ (but only on $\delta, \varepsilon, t$ ), the reachable sets $\vartheta_{f(t)}(h, K(t))$ and $K(t+h)=\vartheta_{f}(h, K(t))$ satisfy for any $h \in[0, T-t]$ $\begin{aligned} d\left(\vartheta_{f(t)}(h, K(t)), \quad K(t+h)\right) & \leq \delta(1+2 B) e^{A h} h, \\ \text { i.e. } \quad \lim _{h \downarrow 0} \frac{1}{h} \cdot d\left(\vartheta_{f(t)}(h, K(t)), \quad K(t+h)\right) & =0 \quad \text { for every } t \in \widetilde{J}_{\varepsilon} .\end{aligned}$

Finally, $\quad f(t) \in \mathcal{F}(K(t))$ for almost every $t \in[0,1[$. Due to Lemma $3.13(\mathrm{e}),(\mathrm{g}),\left.\left.\quad f_{n}(\cdot)\right|_{\widetilde{K}_{j}} \longrightarrow f(\cdot)\right|_{\widetilde{K}_{j}}$ weakly in $L^{1}\left([0,1], C^{0}\left(\widetilde{K}_{j}, \mathbb{R}^{N}\right)\right)$ for each compact set $\widetilde{K}_{j}:=\mathbb{B}_{j+B}\left(K_{0}\right)(j \in \mathbb{N})$ and, $f_{n}(t) \in \mathcal{F}\left(\mathbb{B}_{1 / n}\left(K_{n}(t)\right)\right)$ for every $n \in \mathbb{N}, t \in[0,1[$.

Fixing the index $j \in \mathbb{N}$ of compact sets arbitrarily, Proposition 3.8 provides a sequence $\left(h_{j, n}(\cdot)\right)_{n \in \mathbb{N}}$ with $\quad h_{j, n}(\cdot) \in \operatorname{co}\left\{\left.f_{n}(\cdot)\right|_{\widetilde{K}_{j}},\left.f_{n+1}(\cdot)\right|_{\widetilde{K}_{j}} \ldots\right\} \subset L^{1}\left([0,1], C^{0}\left(\widetilde{K}_{j}, \mathbb{R}^{N}\right)\right)$ such that for $\mathcal{L}^{1}$ almost every $t \in[0,1]$,

$$
\left.h_{j, n}(t) \longrightarrow f(t)\right|_{\widetilde{K}_{j}} \quad(n \longrightarrow \infty) \quad \text { weakly in } C^{0}\left(\widetilde{K}_{j}, \mathbb{R}^{N}\right) .
$$

Proposition 3.10 and assumption (2.) of Viability Theorem 3.11, i.e.

$$
\sup _{M \in \mathcal{K}\left(\mathbb{R}^{N}\right)} \sup _{g \in \mathcal{F}(M)} \operatorname{Lip} g \leq A<\infty, \quad \sup _{M \in \mathcal{K}\left(\mathbb{R}^{N}\right)} \sup _{g \in \mathcal{F}(M)}\|g\|_{\infty} \leq B<\infty,
$$

imply $\left.\quad h_{j, n}(t) \longrightarrow f(t)\right|_{\widetilde{K}_{j}} \quad$ uniformly in $\widetilde{K}_{j} \quad$ for $n \longrightarrow \infty$ and a.e.t $\in[0,1[$. Let $C_{j} \subset[0,1[$ denote the set of full measure for which this uniform convergence holds. Then $C:=\bigcap_{j \in \mathbb{N}} C_{j} \subset\left[0,1\left[\right.\right.$ is also a set of full measure, i.e. $\mathcal{L}^{1}([0,1] \backslash C)=0$.

Choose $t \in C$ arbitrarily. Then for each $j \in \mathbb{N}$, there exists an index $n_{j}>j$ such that $n_{j}>n_{j-1}$ and $\left\|\left.h_{j, n_{j}}(\cdot)\right|_{\widetilde{K}_{j}}-\left.f(\cdot)\right|_{\widetilde{K}_{j}}\right\|_{\infty}<\frac{1}{j}$.

Due to $h_{j, n}(\cdot) \in \operatorname{co}\left\{\left.f_{n}(\cdot)\right|_{\widetilde{K}_{j}},\left.f_{n+1}(\cdot)\right|_{\widetilde{K}_{j}} \ldots\right\}$, each $\left.h_{j, n_{j}}(t)\right|_{\widetilde{K}_{j}}$ has a continuation to $\mathbb{R}^{N}$ in $\operatorname{co}\left\{f_{n}(t), f_{n+1}(t) \ldots\right\} \subset C^{0}\left(\mathbb{R}^{N}, \mathbb{R}^{N}\right.$ ) (that again is denoted by $h_{j, n_{j}}(t)$ ) and, $\quad h_{j, n_{j}}(t) \longrightarrow f(t) \quad$ locally uniformly in $\mathbb{R}^{N} \quad$ for $j \longrightarrow \infty$.

Furthermore, $\quad c o\left\{f_{n}(t), f_{n+1}(t) \ldots\right\} \subset \overline{c o} \mathcal{F}\left(\mathbb{B}_{1 / n}\left(\bigcup_{m \geq n} K_{m}(t)\right)\right)$.

So finally, $d\left(K_{n}(t), K(t)\right) \longrightarrow 0$ and the assumption (3.) about the closed graph of $\mathcal{F}$ (with its convex values) imply $f(t) \in \mathcal{F}(K(t))$. 
4. An application to shape optimization under state constraints. Let $J: \mathcal{K}\left(\mathbb{R}^{N}\right) \longrightarrow \mathbb{R}$ be a shape functional that is Lipschitz continuous with respect to the Pompeiu-Hausdorff distance $d$. Moreover, $\mathcal{V} \subset \mathcal{K}\left(\mathbb{R}^{N}\right)$ denotes a nonempty closed constrained set. Detecting a minimizer $\widehat{K} \in \mathcal{V}$ of the optimization problem $\inf \left\{J(K) \mid K \in \mathcal{V} \subset \mathcal{K}\left(\mathbb{R}^{N}\right)\right\}$

usually proves to be rather complicated $([11,12]$, see $[17,24,38]$ supplementarily). Thus, we prefer here to isolate candidates (for a minimizer) constructively by means of a necessary condition (similarly to [25]). Viability Theorem 3.11 for morphological inclusions is then to lay the basis for a curve $K(\cdot):\left[0, \infty\left[\longrightarrow \mathcal{V} \subset \mathcal{K}\left(\mathbb{R}^{N}\right)\right.\right.$ such that

(i) $\quad t \mapsto J(K(t))$ is nonincreasing and

(ii) every compact set $C=\operatorname{Lim}_{n \rightarrow \infty} K\left(t_{n}\right) \in \mathcal{V}$ (for some sequence $t_{n} \nearrow \infty$ ) satisfies a necessary condition on minimizers (in the form of Fermat's rule).

The first step is to specify a map $\mathcal{F}: \mathcal{K}\left(\mathbb{R}^{N}\right) \rightsquigarrow \operatorname{Lip}\left(\mathbb{R}^{N}, \mathbb{R}^{N}\right)$ satisfying the following conditions on its values:

1.) all values of $\mathcal{F}$ are convex and closed (w.r.t. locally uniform convergence),

2.) $\sup _{M \in \mathcal{K}\left(\mathbb{R}^{N}\right)} \sup _{f \in \mathcal{F}(M)}\left(\|f\|_{\infty}+\operatorname{Lip} f\right)<\infty$,

Essentially, the choice of $\mathcal{F}$ is to guarantee that the composition $t \mapsto J(K(t))$ is nonincreasing for every compact-valued solution $K(\cdot):[0,1] \rightsquigarrow \mathbb{R}^{N}$ of the morphological inclusion $\stackrel{\circ}{K}(\cdot) \cap \mathcal{F}(K(\cdot)) \neq \emptyset$. For combining this aim with the conditions on its values, we do not use the so-called shape semiderivative of $J$ in direction $v(\cdot)$

$$
\delta J(K)(v):=\lim _{h \downarrow 0} \frac{1}{h} \cdot\left(J\left(\vartheta_{v}(h, K)\right)-J(K)\right)
$$

for $K \in \mathcal{K}\left(\mathbb{R}^{N}\right)$ (assuming the limit to exist) as in [11, 12], but we prefer the notion of Clarke's generalized directional derivative in a Banach space (see e.g. [10]) and extend it to shape transitions:

Definition 4.1. Let $J:\left(\mathcal{K}\left(\mathbb{R}^{N}\right), d l\right) \longrightarrow \mathbb{R}$ be a Lipschitz continuous shape functional. Clarke's generalized shape derivative of $J(\cdot)$ at $K \in \mathcal{K}\left(\mathbb{R}^{N}\right)$ in direction $v \in \operatorname{Lip}\left(\mathbb{R}^{N}, \mathbb{R}^{N}\right)$ is defined as

$$
\begin{gathered}
\delta^{C} J(K)(v):=\limsup _{\substack{h \downarrow 0, M \rightarrow K \\
\left(M \in \mathcal{K}\left(\mathbb{R}^{N}\right)\right)}} \frac{1}{h} \cdot\left(J\left(\vartheta_{v}(h, M)\right)-J(M)\right) . \\
\text { Set } \iota J(K):=\limsup _{\substack{M \rightarrow K_{K}^{K} \\
\left(M \in \mathcal{K}\left(\mathbb{R}^{N}\right)\right)}} \inf \left\{\delta^{C} J(M)(v) \mid v \in \operatorname{Lip}\left(\mathbb{R}^{N}, \mathbb{R}^{N}\right),\|v\|_{\infty}+\operatorname{Lip} v \leq 1\right\} .
\end{gathered}
$$

Remark. $\quad$ 1. Let $\Lambda \geq 0$ denote the Lipschitz constant of $J:\left(\mathcal{K}\left(\mathbb{R}^{N}\right), d l\right) \longrightarrow \mathbb{R}$. Then, due to Lemma 2.4, $\left|J\left(\vartheta_{v}(h, K)\right)-J(K)\right| \leq \Lambda \cdot d l\left(\vartheta_{v}(h, K), K\right) \leq \Lambda \cdot\|v\|_{\infty} h$ for every $v \in \operatorname{Lip}\left(\mathbb{R}^{N}, \mathbb{R}^{N}\right)$ and thus, $\quad\left|\delta^{C} J(K)(v)\right| \leq \Lambda\|v\|_{\infty}, \quad \iota J(\bar{K}) \geq-\Lambda$. In particular, $\delta^{C} J(K)(0)=0$ for every $K \in \mathcal{K}\left(\mathbb{R}^{N}\right)$.

2. $\iota J(\cdot): \mathcal{K}\left(\mathbb{R}^{N}\right) \longrightarrow \mathbb{R}$ is the upper semicontinuous envelope of the minimal generalized shape derivative $\delta^{C} J(\cdot)(v)$ over all $v$ in the unit ball of $\operatorname{Lip}\left(\mathbb{R}^{N}, \mathbb{R}^{N}\right)$. Furthermore, $\iota J(K) \leq \delta^{C} J(K)(0)=0$ for all $K \in \mathcal{K}\left(\mathbb{R}^{N}\right)$.

DEFINITION 4.2. Using the notation of Def. 4.1, set $\mathcal{F}: \mathcal{K}\left(\mathbb{R}^{N}\right) \rightsquigarrow \operatorname{Lip}\left(\mathbb{R}^{N}, \mathbb{R}^{N}\right)$, $\mathcal{F}(K):=\left\{v \in \operatorname{Lip}\left(\mathbb{R}^{N}, \mathbb{R}^{N}\right) \mid\|v\|_{\infty}+\operatorname{Lip} v \leq 1, \quad \delta^{C} J(K)(v) \leq \frac{1}{2} \cdot \iota J(K)\right\}$. 
Here both the bound 1 and the factor $\frac{1}{2}$ are rather arbitrary. We show in subsequent Lemma 4.5 that all values of the set-valued map $\mathcal{F}$ are nonempty, convex and closed with respect to locally uniform convergence.

Lemma 4.3. Let $J:\left(\mathcal{K}\left(\mathbb{R}^{N}\right), d l\right) \longrightarrow \mathbb{R}$ be a Lipschitz continuous shape functional and $K(\cdot):[0,1] \rightsquigarrow \mathbb{R}^{N}$ be any compact-valued Lipschitz solution of the morphological inclusion $\stackrel{\circ}{K}(\cdot) \cap \mathcal{F}(K(\cdot)) \neq \emptyset$ with $\mathcal{F}(\cdot)$ introduced in Def. 4.2. Then,

1. $[0,1] \longrightarrow \mathbb{R}, t \longmapsto J(K(t))$ is nonincreasing.

2. Suppose $\inf _{\mathcal{K}\left(\mathbb{R}^{N}\right)} J(\cdot)>-\infty$ in addition. and, let $C$ belong to the $\omega$-limit set of $K(\cdot)$ in $\mathcal{K}\left(\mathbb{R}^{N}\right)$, i.e. $d\left(K\left(t_{n}\right), C\right) \longrightarrow 0$ for some $t_{n} \nearrow \infty$. Then $\iota J(C)=0$.

Proof. 1. results from the Lipschitz continuity of the composition $[0,1] \longrightarrow \mathbb{R}$, $t \longmapsto J(K(t))$ and the definition of $\mathcal{F}$. Indeed, at almost every time $t \in[0,1]$, there exists $v \in \mathcal{F}(K(t)) \subset \operatorname{Lip}\left(\mathbb{R}^{N}, \mathbb{R}^{N}\right)$ with $\lim _{h \downarrow 0} \frac{1}{h} \cdot d l\left(K(t+h), \vartheta_{v}(h, K(t))\right)=0$.

Thus,

$$
\begin{array}{lll}
\limsup _{h \downarrow 0} \frac{1}{h} \cdot(J(K(t+h))-J(K(t))) & \\
\leq & \limsup _{h \downarrow 0} \frac{1}{h} \cdot\left(J\left(\vartheta_{v}(h, K(t))\right)-J(K(t))+\right. & \left.\operatorname{Lip} J \cdot d\left(\vartheta_{v}(h, K(t)), K(t+h)\right)\right) \\
\leq & \delta^{C} J(K(t))(v) & \\
\leq & \frac{1}{2} \cdot \iota J(K(t)) & \text { (since } v \in \mathcal{F}(K(t))) \\
\leq & 0 & \text { (due to preceding Remark }(2 .)) .
\end{array}
$$

2. Assume the contrary, i.e. $\kappa:=\iota J(C)<0$. Then, there exists some small $\rho>0$ such that all sets $M \in \mathcal{K}\left(\mathbb{R}^{N}\right)$ with $d(M, C) \leq 2 \rho$ satisfy $\iota J(M)<\frac{\kappa}{2}<0$. For all $n \in \mathbb{N}$ sufficiently large, $K\left(t_{n}\right)$ has the Pompeiu-Hausdorff distance $<\rho$ from $C$. As all values of $\mathcal{F}$ contain only functions $v \in \operatorname{Lip}\left(\mathbb{R}^{N}, \mathbb{R}^{N}\right)$ with $\|v\|_{\infty}+\operatorname{Lip} v \leq 1$, every compact-valued solution $K(\cdot)$ of the morphological inclusion $\stackrel{\circ}{K}(\cdot) \cap \mathcal{F}(K(\cdot)) \neq \emptyset$ has Lipschitz constant $\leq 1$ (with respect to $d$ ) similarly to Lemma 2.4.

Thus, $d(K(s), C)<2 \rho$ for all $s \in\left[t_{n}-\rho, t_{n}+\rho\right]$. Now the same adaptation of chain rule as in (1.) implies $J\left(K\left(s_{2}\right)\right) \leq J\left(K\left(s_{1}\right)\right)+\frac{\kappa}{4} \cdot\left(s_{2}-s_{1}\right)$ for all $t_{n}-\rho \leq s_{1} \leq s_{2} \leq t_{n}+\rho$ and large $n \in \mathbb{N}$ - contradicting the hypothesis inf $J(\cdot)>-\infty$.

The next lemma will be used for verifying the convexity of all values of $\mathcal{F}$ in Lemma 4.5:

Lemma 4.4. For every $\lambda \in] 0,1\left[\right.$, there exists $\mu \in L^{1}([0,1])$ satisfying $\frac{1}{t} \cdot \int_{0}^{t}(\mu(s)-\lambda) d s \longrightarrow 0 \quad(t \downarrow 0), \quad \mu(\cdot) \in\{0,1\}$ piecewise constant in $] 0,1[$.

Proof. $\mu(\cdot)$ is defined in each interval $\left[\frac{1}{\sqrt{n+1}}, \frac{1}{\sqrt{n}}[(n \in \mathbb{N})\right.$.

Set $\quad \mu(t):=\left\{\begin{aligned} 0 & \text { for } & \frac{1}{\sqrt{n+1}} \leq t<\frac{\lambda}{\sqrt{n+1}}+\frac{1-\lambda}{\sqrt{n}} \\ 1 & \text { for } \quad \frac{\lambda}{\sqrt{n+1}}+\frac{1-\lambda}{\sqrt{n}} & \leq t<\frac{1}{\sqrt{n}}\end{aligned} \quad\right.$ for each $n \in \mathbb{N}$.

Then, $\quad \int_{\frac{1}{\sqrt{n+1}}}^{\frac{1}{\sqrt{n}}}(\mu(s)-\lambda) d s=0$ and thus, $\int_{0}^{\frac{1}{\sqrt{n}}}(\mu(s)-\lambda) d s=0$.

Moreover, $\quad \int_{\frac{1}{\sqrt{n+1}}}^{\frac{1}{\sqrt{n}}}|\mu(s)-\lambda| \quad d s=2 \lambda(1-\lambda)\left(\frac{1}{\sqrt{n}}-\frac{1}{\sqrt{n+1}}\right) \quad$ implies

$\sup _{\frac{1}{\sqrt{n+1}} \leq t \leq \frac{1}{\sqrt{n}}} \frac{1}{t} \cdot\left|\int_{0}^{t}(\mu(s)-\lambda) d s\right| \leq \sqrt{n+1} \cdot \int_{\frac{1}{\sqrt{n+1}}}^{\frac{1}{\sqrt{n}}}|\mu(s)-\lambda| d s \stackrel{n \rightarrow \infty}{\longrightarrow} 0$. 
LEMma 4.5. Consider $\operatorname{Lip}\left(\mathbb{R}^{N}, \mathbb{R}^{N}\right)$ with the topology of locally uniform convergence. All values of the set-valued map $\mathcal{F}: \mathcal{K}\left(\mathbb{R}^{N}\right) \rightsquigarrow \operatorname{Lip}\left(\mathbb{R}^{N}, \mathbb{R}^{N}\right)$ introduced in Definition 4.2 are nonempty, convex and closed.

Proof. For every $K \in \mathcal{K}\left(\mathbb{R}^{N}\right)$, the value $\mathcal{F}(K)$ is a nonempty because either $\quad \iota J(K)=0 \quad$ and then, $0 \in \mathcal{F}(K)$

or $\quad \iota J(K)<0 \quad$ and then there is $v \in \operatorname{Lip}\left(\mathbb{R}^{N}, \mathbb{R}^{N}\right)$ with $\|v\|_{\infty}+\operatorname{Lip} v \leq 1$, $\delta^{C} J(K)(v) \leq \frac{3}{4} \cdot \iota J(K)<0$ (due to the def. of infimum), i.e. $v \in \mathcal{F}(K)$ induces a shape transition along which $J(\cdot)$ is strictly decreasing for short times.

Furthermore, $\mathcal{F}(K) \subset \operatorname{Lip}\left(\mathbb{R}^{N}, \mathbb{R}^{N}\right)$ is convex. Indeed, choose any $v, w \in \mathcal{F}(K)$ and $\lambda \in] 0,1\left[\right.$. According to Lemma 4.4, there exists some $\mu \in L^{1}([0,1])$ satisfying $\frac{1}{t} \cdot \int_{0}^{t}(\mu(s)-\lambda) d s \longrightarrow 0 \quad(t \downarrow 0), \quad \mu(\cdot) \in\{0,1\}$ piecewise constant in $] 0,1[$. Now we compare the evolution of an arbitrary set $K \in \mathcal{K}\left(\mathbb{R}^{N}\right)$ along the autonomous Lipschitz field

$$
u: \mathbb{R}^{N} \quad \longrightarrow \mathbb{R}^{N}, \quad x \longmapsto \lambda \cdot v(x)+(1-\lambda) \quad \cdot w(x)
$$

and along the nonautonomous vector field

$$
g: \quad \mathbb{R}^{N} \times[0,1] \longrightarrow \mathbb{R}^{N}, \quad(x, t) \longmapsto \mu(t) \cdot v(x)+(1-\mu(t)) \cdot w(x) .
$$

We verify $d\left(\vartheta_{u}(t, M), \vartheta_{g}(t, M)\right) \leq o(t)$ for $t \downarrow 0$ uniformly in $M$.

For any initial point $x_{0} \in \mathbb{R}^{N}$ given, let $x(\cdot) \in C^{1}\left([0,1], \mathbb{R}^{N}\right)$ denote the (unique) solution to $x^{\prime}(\cdot)=u(x(\cdot)), \quad x(0)=x_{0}$. As each $g(\cdot, t)$ is Lipschitz continuous (with Lipschitz constant $\leq 1$ ), Filippov's Theorem (applied to differential equations here) guarantees that the Cauchy problem

$$
\left\{\begin{array}{l}
y^{\prime}(\cdot)=g(y(\cdot), \cdot) \quad \text { almost everywhere in }[0,1] \\
y(0)=x_{0} \in \mathbb{R}^{N}
\end{array}\right.
$$

has a solution $y(\cdot) \in W^{1,1}\left([0, t], \mathbb{R}^{N}\right)$. These solutions $x(\cdot), y(\cdot)$ always satisfy

$$
\begin{aligned}
&|x(t)-y(t)| \\
&=\mid \int_{0}^{t}(\lambda v(x(s))-\mu(s) v(y(s))+(1-\lambda) w(x(s))-(1-\mu(s)) w(y(s))) d s \mid \\
& \leq\left|\int_{0}^{t}((\lambda-\mu(s)) v(x(s)) \quad+(\mu(s)-\lambda) w(x(s))) d s\right| \\
&+\int_{0}^{t} \mu(s) \cdot \operatorname{Lip} v \cdot|x(s)-y(s)| d s \quad+\int_{0}^{t}(1-\mu(s)) \cdot \operatorname{Lip} w \cdot|x(s)-y(s)| d s \\
& \leq\left|\int_{0}^{t}(\lambda-\mu(s)) \cdot\left(v\left(x_{0}\right)-w\left(x_{0}\right)\right) d s\right|+\int_{0}^{t}|\lambda-\mu(s)|(\operatorname{Lip} v+\operatorname{Lip} w)\left|x(s)-x_{0}\right| d s \\
& \leq 2\left|\int_{0}^{t}(\lambda-\mu(s)) d s\right|+\int_{0}^{t} 1 \cdot 2 \cdot s \text { ds }+\int_{0}^{t}|x(s)-y(s)| d s
\end{aligned}
$$

due to $\|v\|_{\infty}+\operatorname{Lip} v \leq 1,\|w\|_{\infty}+\operatorname{Lip} w \leq 1$.

Gronwall's Lemma ensures $|x(t)-y(t)| \leq o(t)$ for $t \downarrow 0$ uniformly in $x_{0} \in \mathbb{R}^{N}$. (In particular, the estimate of Filippov's Theorem is difficult to be applied here immediately as the integral mean of $\mu(\cdot)-\lambda$, but not of $|\mu(\cdot)-\lambda|$ is $o(t)$ for $t \downarrow 0$.) Thus, for any initial set $M \in \mathcal{K}\left(\mathbb{R}^{N}\right)$, the reachable sets satisfy

$$
\operatorname{dist}\left(\vartheta_{u}(t, M), \vartheta_{g}(t, M)\right) \leq o(t) \quad \text { for } t \downarrow 0 \quad \text { uniformly in } M \in \mathcal{K}\left(\mathbb{R}^{N}\right) .
$$


The same uniform estimates holds for $\operatorname{dist}\left(\vartheta_{g}(t, M), \vartheta_{u}(t, M)\right)$ since the preceding solutions $x(\cdot)$ and $y(\cdot)$ have needed only a joint initial point $x_{0} \in \mathbb{R}^{N}$.

According to the proof of Lemma 4.4, we can suppose to have a sequence $s_{n} \searrow 0$ in $] 0,1\left[\right.$ such that $\mu(\cdot) \in\{0,1\}$ is constant in $\left[s_{n+1}, s_{n}\right.$ [ for each $n \in \mathbb{N}$. So for every set $M \in \mathcal{K}\left(\mathbb{R}^{N}\right)$ and time $t \in\left[s_{n+1}, s_{n}\right]$, the reachable set $\vartheta_{g}(t, M)$ is either $\vartheta_{v}\left(t-s_{n+1}, \vartheta_{g}\left(s_{n+1}, M\right)\right)$ or $\vartheta_{w}\left(t-s_{n+1}, \vartheta_{g}\left(s_{n+1}, M\right)\right)$.

Fix $\varepsilon>0$ arbitrarily small. For each $\left.h \in] 0, s_{1}\right]$, choose $m \in \mathbb{N}$ with $s_{m}<h \leq s_{m-1}$ and, we obtain (at least) for small $h>0$ and $M \in \mathcal{K}\left(\mathbb{R}^{N}\right)$ sufficiently close to $K$

$J\left(\vartheta_{u}(h, M)\right)-J(M)$

$\leq J\left(\vartheta_{g}(h, M)\right)-J(M)$

$+o(h)$

$=J\left(\vartheta_{g}(h, M)\right)-J\left(\vartheta_{g}\left(s_{m}, M\right)\right)+\sum_{n=m}^{\infty}\left(J\left(\vartheta_{g}\left(s_{n}, M\right)\right)-J\left(\vartheta_{g}\left(s_{n+1}, M\right)\right)\right)+o(h)$

$\leq\left(h-s_{m}\right) \cdot\left(\frac{\iota J(K)}{2}+\varepsilon\right) \quad+\sum_{n=m}^{\infty}\left(s_{n}-s_{n+1}\right) \cdot\left(\frac{\iota J(K)}{2}+\varepsilon\right) \quad+o(h)$

$=h \cdot\left(\frac{\iota J(K)}{2}+\varepsilon\right)$

$+o(h)$

So, $\delta^{C} J(K)(u) \leq \frac{\iota J(K)}{2}+\varepsilon$ with any $\varepsilon>0$, i.e. $u \stackrel{\text { Def. }}{=} \lambda v+(1-\lambda) w \in \mathcal{F}(K)$.

Finally, we verify that $\mathcal{F}(K)$ is closed. Let $\left(v_{n}\right)_{n \in \mathbb{N}}$ be a sequence in $\mathcal{F}(K) \subset$ $\operatorname{Lip}\left(\mathbb{R}^{N}, \mathbb{R}^{N}\right)$ converging to $v \in \operatorname{Lip}\left(\mathbb{R}^{N}, \mathbb{R}^{N}\right)$ locally uniformly. Obviously, the limit holds $\|v\|_{\infty}+\operatorname{Lip} v \leq 1$. Moreover, similarly to Lemma 2.4, Filippov's Theorem implies

$$
\sup _{\substack{M \in \mathcal{K}\left(\mathbb{R}^{N}\right) \\ d(K, M) \leq 1}} d\left(\vartheta_{v_{n}}(h, M), \vartheta_{v}(h, M)\right) \leq h e^{h} \cdot \sup _{\mathbb{B}_{2}(K)}\left|v_{n}(\cdot)-v(\cdot)\right| \longrightarrow 0
$$

for $n \longrightarrow \infty$ uniformly in $h \in[0,1]$ and for every set $K \in \mathcal{K}\left(\mathbb{R}^{N}\right)$. So due to the Lipschitz continuity of $J(\cdot)$, Clarke's generalized shape derivative satisfies

$$
\begin{aligned}
\delta^{C} J(K)(v) \leq \limsup _{\substack{h \downarrow 0, M \rightarrow K \\
\left(M \in \mathcal{K}\left(\mathbb{R}^{N}\right)\right)}} \frac{1}{h} \cdot\left(J\left(\vartheta_{v_{n}}(h, M)\right)-J(M)\right) & +\operatorname{Lip} J \cdot \sup _{\mathbb{B}_{2}(K)}\left|v_{n}(\cdot)-v(\cdot)\right| \\
& \leq \frac{1}{2} \cdot \iota J(K) \\
& +\operatorname{Lip} J \cdot \sup _{\mathbb{B}_{2}(K)}\left|v_{n}(\cdot)-v(\cdot)\right|
\end{aligned}
$$

$n \longrightarrow \infty$ reveals $v \in \mathcal{F}(K)$.

Remark. In regard to Viability Theorem 3.11, the graph of $\mathcal{F}: \mathcal{K}\left(\mathbb{R}^{N}\right) \rightsquigarrow \operatorname{Lip}\left(\mathbb{R}^{N}, \mathbb{R}^{N}\right)$ ought to be closed (still using the topology of locally uniform convergence). This feature is closely related with the lower semicontinuity of $\delta^{C} J(\cdot)(v): \mathcal{K}\left(\mathbb{R}^{N}\right) \longrightarrow \mathbb{R}$ (with $v \in \operatorname{Lip}\left(\mathbb{R}^{N}, \mathbb{R}^{N}\right)$ fixed) and, it will be dealt here as an additional assumption about $J$.

So now Lemmas 4.3 and 4.5 have laid the basis for applying the morphological Viability Theorem 3.11. We summarize the main result of this paragraph:

Proposition 4.6. $\quad$ Suppose $J: \mathcal{K}\left(\mathbb{R}^{N}\right) \longrightarrow \mathbb{R}$ to be Lipschitz continuous with respect to the Pompeiu-Hausdorff distance $d$ and bounded from below.

Using Definitions 4.1, 4.2, $\mathcal{F}: \mathcal{K}\left(\mathbb{R}^{N}\right) \rightsquigarrow \operatorname{Lip}\left(\mathbb{R}^{N}, \mathbb{R}^{N}\right)$ is assumed to have closed graph with respect to locally uniform convergence on $\operatorname{Lip}\left(\mathbb{R}^{N}, \mathbb{R}^{N}\right)$.

Let $\mathcal{V} \subset \mathcal{K}\left(\mathbb{R}^{N}\right)$ be nonempty and closed such that for every $K \in \mathcal{V}$, the intersection $\mathcal{F}(K) \cap T_{\mathcal{V}}(K)$ is nonempty. 
Then there exists a Lipschitz continuous solution $K:\left[0, \infty\left[\longrightarrow \mathcal{V} \subset \mathcal{K}\left(\mathbb{R}^{N}\right)\right.\right.$ of the morphological inclusion $\stackrel{\circ}{K}(\cdot) \cap \mathcal{F}(K(\cdot)) \neq \emptyset$ such that

1. $[0, \infty[\longrightarrow \mathbb{R}, t \longmapsto J(K(t))$ is nonincreasing,

2. every element $C \in \mathcal{K}\left(\mathbb{R}^{N}\right)$ of its $\omega$-limit set in $\mathcal{K}\left(\mathbb{R}^{N}\right)$ satisfies the following

necessary condition on minimizers of $J(\cdot)$ in $\mathcal{K}\left(\mathbb{R}^{N}\right): \quad \iota J(C)=0$.

Acknowledgments. The author would like to thank Irina Surovtsova and Daniel Andrej for fruitful complementary discussions. He is also grateful to the anonymous referees since their very detailed reports contributed a lot to improving this article.

\section{REFERENCES}

[1] Artstein, Z. (1995): A calculus for set-valued maps and set-valued evolution equations, Set-Valued Anal. 3, No.3, pp. 213-261

[2] Aubin, J.-P. (1999): Mutational and Morphological Analysis: Tools for Shape Evolution and Morphogenesis, Birkhäuser, Systems and Control: Foundations and Applications

[3] Aubin, J.-P. (1993): Mutational equations in metric spaces, Set-Valued Analysis 1, pp. 3-46

[4] Aubin, J.-P. (1992): A note on differential calculus in metric spaces and its applications to the evolution of tubes, Bull. Pol. Acad. Sci., Math. 40, No.2, pp. 151-162

[5] Aubin, J.-P. (1991) : Viability Theory, Birkhäuser, Systems and Control: Foundations and Applications

[6] Aubin, J.-P. \& Cellina, A. (1984): Differential Inclusions, Springer, Grundlehren der mathematischen Wissenschaften 264

[7] Aubin, J.-P. \& Frankowska, H. (1990): Set-Valued Analysis, Birkhäuser, Systems and Control : Foundations and Applications

[8] Bárcenas, D. (2001): Weak compactness criteria for set-valued integrals and RadonNikodym theorem for vector-valued multimeasures, Czech. Math. J. 51 (126), pp. 493504

[9] Céa, J. (1976): Une méthode numérique pour la recherche d"un domaine optimal, in: Glowinski, R. \& Lions, J.L. (Eds.), Computing methods in applied sciences and engineering. Part 1, Springer, Lecture Notes in Economics and Mathematical Systems 134, pp. 245-257

[10] Clarke, F.H. (1983) : Optimization and Nonsmooth Analysis, Wiley-Interscience, Canadian Mathematical Society Series of Monographs and Advanced Texts

[11] Delfour, M. \& Zolésio, J.-P. (2001): Shapes and Geometries: Analysis, Differential Calculus and Optimization, SIAM, Advances in Design and Control

[12] Delfour, M. \& Zolésio, J.-P. (1991): Velocity method and Lagrangian formulation for the computation of the shape Hessian, SIAM J. Control Optim. 29, No. 6, pp. 1414-1442

[13] Diestel, J. (1977): Remarks on weak compactness in $L^{1}(\mu, X)$, Glasgow Math. J. 18, pp. 8791

[14] Diestel, J., Ruess, W.M. \& Schachermeyer, W. (1993): Weak compactness in $L^{1}(\mu, X)$, Proc. Amer. Math. Soc. 118, No.2, pp. 443-453

[15] Diestel, J. \& Uhl, J. (1977): Vector measures, Math. Surveys vol. 15, Amer. Math. Soc.

[16] Doyen, L. (1995): Mutational equations for shapes and vision-based control, J. Math. Imaging Vis. 5, No.2, pp. 99-109

[17] Doyen, L. (1994): Inverse function theorems and shape optimization, SIAM J. Control Optimization 32, No.6, pp. 1621-1642

[18] Doyen, L. (1994): Shape Lyapunov functions and stabilization of reachable tubes of control problems. J. Math. Anal. Appl. 184, No.2, pp. 222-228

[19] Doyen, L. (1993): Filippov and invariance theorems for mutational inclusions of tubes, Set-Valued Anal. 1, No.3, pp. 289-303

[20] Frankowska, H., Plaskacz, S. \& Rzeżuchowski, T.: Measurable viability theorems and the Hamilton-Jacobi-Bellman equation, J. Differ. Equations 116, No.2 (1995), 265-305

[21] Green, J.W. \& Valentine, F.A. (1960/61): On the Arzel-Ascoli theorem, Math. Mag. 34, pp. $199-202$

[22] Gorre, A. (1997): Evolutions of tubes under operability constraints. J. Math. Anal. Appl. 216, No.1, pp. 1-22 
[23] Kisielewicz, M. (1992): Weak compactness in spaces $C(S, X)$, in: Information theory, statistical decision functions, random processes, Trans. 11th Prague Conf., Prague/Czech. 1990, Vol. B, pp. 101-106

[24] Lorenz, Th. (2006) : Reynold's transport theorem for differential inclusions, Set-Valued Analysis 14, No.3, pp. 209-247

[25] Lorenz, Th. (2001): Set-valued maps for image segmentation, Comput. Vis. Sci. 4, No.1, pp. $41-57$

[26] Nagumo, M. (1942): Über die Lage der Integralkurven gewöhnlicher Differentialgleichungen, Proc. Phys.-Math. Soc. Japan, III. Ser. 24, pp. 551-559

[27] Panasyuk, A.I. (1995): Quasidifferential equations in a complete metric space under conditions of the Carathéodory type. I, Differ. Equations 31, No.6, pp. 901-910

[28] Panasyuk, A.I. (1992): Properties of solutions of a quasidifferential approximation equation and the equation of an integral funnel, Differ. Equations 28, No.9, pp. 1259-1266

[29] Panasyuk, A.I (1985): Quasidifferential equations in metric spaces, Differ. Equations 21, pp. $914-921$

[30] Quincampoix, M. \& Veliov, V. (1999): Open-loop viable control under uncertain initial state information. Set-Valued Anal. 7, No.1, pp. 55-87

[31] Ricceri, B. \& Villani, A. (1983): Separability and Scorza-Dragoni's property, Le Matematiche, 37, No.1, pp. 156-161

[32] Scorza-Dragoni, G. (1948): Un teorema sulle funzioni continue rispetto ad una e misurabili rispetto ad un'altra variabile, Rend. Semin. Mat. Univ. Padova, 17, pp. 102-108

[33] Sokolowski, J. \& Zolésio, J.-P. (1992): Introduction to Shape Optimization. Shape Sensitivity Analysis, Springer, Series in Computational Mathematics 16

[34] Ülger, A. (1991): Weak compactness in $L^{1}(\mu, X)$, Proc. Amer. Math. Soc. 113, No.1, pp. $143-149$

[35] Vinter, R. (2000): Optimal Control, Birkhäuser, Systems and Control: Foundations and Applications

[36] Yosida, K. (1978): Functional Analysis, fifth edition, Springer, Grundlehren der mathematischen Wissenschaften 123

[37] Ziemer, W. (1989): Weakly Differentiable Functions, Springer, Graduate texts in mathematics 120

[38] Zolésio, J.-P. (1979): Identification de domaine par déformations. Thèse de doctorat d'état, Université de Nice 